MATHEMATICS OF COMPUTATION

Volume 73, Number 248, Pages 1673-1697

S 0025-5718(03)01615-6

Article electronically published on October 27, 2003

\title{
VORTICITY-VELOCITY-PRESSURE FORMULATION FOR STOKES PROBLEM
}

\author{
M. AMARA, E. CHACÓN VERA, AND D. TRUJILLO
}

\begin{abstract}
We propose a three-field formulation for efficiently solving a twodimensional Stokes problem in the case of nonstandard boundary conditions. More specifically, we consider the case where the pressure and either normal or tangential components of the velocity are prescribed at some given parts of the boundary. The proposed computational methodology consists in reformulating the considered boundary value problem via a mixed-type formulation where the pressure and the vorticity are the principal unknowns while the velocity is the Lagrange multiplier. The obtained formulation is then discretized and a convergence analysis is performed. A priori error estimates are established, and some numerical results are presented to highlight the perfomance of the proposed computational methodology.
\end{abstract}

\section{INTRODUCTION}

We consider in this work the stationary Stokes equations with nonstandard boundary conditions in a bounded domain $\Omega \subset \mathbb{R}^{2}$, with a polygonal boundary $\Gamma=\partial \Omega$. $\Omega$ is assumed to be on one side of the boundary $\Gamma$. The velocity field $\mathbf{u}=\left(u_{1}, u_{2}\right)^{t}$ and the pressure $p$ satisfy

$$
\begin{aligned}
-\nu \Delta \mathbf{u}+\nabla p & =\mathbf{f} \text { in } \Omega, \\
\operatorname{div} \mathbf{u} & =0 \text { in } \Omega,
\end{aligned}
$$

where $\nu>0$ is the kinematic viscosity of the fluid and $\mathbf{f}$ is the density of external forces. Our aim is to adopt a three-field formulation involving the velocity, the pressure and the vorticity. This approach is based on a mixed formulation where the principal unknowns are the pressure and the vorticity and the Lagrange multiplier is the velocity. A discrete model associated to this formulation by conforming finite elements is not appropriate due to the lake of coercivity of the discrete formulation. Specifically, the pressure is not well defined if we do not use compatible discrete spaces for the pressure and the velocity. We propose to add a stabilization term in the discrete mixed formulation to restore the coercivity of the form and therefore the well posedness of the discrete problem. This stabilization form consists of the jumps of the discrete vorticity and pressure on the internal edges of the triangulation. This idea has already been used in [12, among other works. We prove that the method is unconditionally convergent in the sense that it does not require additional regularity assumptions. We present in this paper the case where finite elements of degree 1 are used. The description of the general case of finite elements of degree $k$ can be

Received by the editor January 10, 2002 and, in revised form, March 5, 2003.

2000 Mathematics Subject Classification. Primary 65N12; Secondary 35Q30.

(C)2003 American Mathematical Society 
found in [3]. We prove that the method is optimal in terms of finite elements, i.e., we obtain an $O\left(h^{k}\right)$ error estimate when we use finite elements of degree $k$.

The numerical results presented in this paper demonstrate the efficiency of the method by using only simple finite elements (continuous, piecewise of degree 1 for the velocity and constant discontinuous for the pressure and the vorticity). This method can be easily extended to the three-dimensional case. The extension to the Navier-Stokes case is still under investigation.

Throughout this paper, we adopt the following nomenclature and assumptions: For any $2 D$ vector field $\mathbf{v}=\left(v_{1}, v_{2}\right)^{t}$, we use the divergence and scalar rotational operators $\operatorname{div} \mathbf{v}=\partial_{1} v_{1}+\partial_{2} v_{2}$ and $\operatorname{curl} \mathbf{v}=\partial_{1} v_{2}-\partial_{2} v_{1}$, and the vector rotational of any scalar field $\phi, \operatorname{curl} \phi=\left(\partial_{2} \phi,-\partial_{1} \phi\right)^{t}$.

Finally, we recall that for any $2 D$ vector field $\mathbf{v}$, the identity $\nabla \operatorname{div} \mathbf{v}-\operatorname{curl} \operatorname{curl} \mathbf{v}$ $=\Delta \mathbf{v}$ is satisfied.

We suppose that $\Gamma$ is formed by three open and disjoint subsets $\Gamma_{1}, \Gamma_{2}, \Gamma_{3}$ such that $\Gamma=\bar{\Gamma}_{1} \cup \bar{\Gamma}_{2} \cup \bar{\Gamma}_{3}$. Each of the $\Gamma_{i}$ itself might be formed by a set of linear segments, and we denote by $\left\{\mathbf{c}_{i}\right\}$ the vertices of $\Omega$ and by $\left\{\alpha_{i}\right\}$ the openings of the angles of $\Omega$ at each of the $\mathbf{c}_{i}$. We assume $\alpha_{i}<2 \pi$ for each $i$, and we denote by $\mathbf{a}_{j}$, for $j=1, \ldots, l$, the nonconvex corners of $\Omega$, i.e., the corners where $\alpha_{j}>\pi$. We also assume that there are no nonconvex corners at the intersection of $\bar{\Gamma}_{1} \cup \bar{\Gamma}_{2}$ and $\bar{\Gamma}_{3}$.

Introduce the scalar vorticity $\omega=\operatorname{curl} \mathbf{u}$, the outward normal vector $\mathbf{n}$ and the tangent vector $\mathbf{t}$ to the boundary $\Gamma$. Given vector data $\mathbf{u}_{0}, \mathbf{a}, \mathbf{b}$ and scalar data $p_{0}$, $\omega_{0}$, we consider the following boundary conditions:

$$
\begin{array}{llll}
\mathbf{u} \cdot \mathbf{n}=\mathbf{u}_{\mathbf{0}} \cdot \mathbf{n}, & \mathbf{u} \cdot \mathbf{t}=\mathbf{u}_{\mathbf{0}} \cdot \mathbf{t} & \text { on } & \Gamma_{1} \\
\mathbf{u} \cdot \mathbf{t}=\mathbf{a} \cdot \mathbf{t}, & p=p_{0} & \text { on } & \Gamma_{2}, \\
\mathbf{u} \cdot \mathbf{n}=\mathbf{b} \cdot \mathbf{n}, & \omega=\omega_{0} & \text { on } & \Gamma_{3},
\end{array}
$$

together with a compatibility condition for these boundary data, that is, there exists at least one incompressible velocity field which satisfies them, i.e., there exists a function $\mathbf{U}_{0} \in \mathbb{L}^{2}(\Omega)$ with $\operatorname{curl} \mathbf{U}_{0} \in L^{2}(\Omega)$ such that

$$
\operatorname{div} \mathbf{U}_{\mathbf{0}}=0 \text { in } \Omega \text { and }\left\{\begin{array}{lll}
\mathbf{U}_{\mathbf{0}}=\mathbf{u}_{\mathbf{0}} & \text { on } & \Gamma_{1}, \\
\mathbf{U}_{\mathbf{0}} \cdot \mathbf{t}=\mathbf{a} \cdot \mathbf{t} & \text { on } & \Gamma_{2}, \\
\mathbf{U}_{\mathbf{0}} \cdot \mathbf{n}=\mathbf{b} \cdot \mathbf{n} & \text { on } & \Gamma_{3}
\end{array}\right.
$$

Therefore, we will work with homogeneous boundary conditions, i.e., $\mathbf{u}_{0}=$ 0 on $\Gamma_{1}, \mathbf{a}=0$ on $\Gamma_{2}$ and $\mathbf{b}=0$ on $\Gamma_{3}$.

To illustrate the above boundary conditions we can consider a pipe flow problem where we impose simultaneously this family of boundary conditions (see [7]):

\begin{tabular}{|ccc|}
\hline Boundary & Conditions & Duct flow application \\
\hline$\Gamma_{1}$ & $\mathbf{u}=0$ or $\mathbf{u}=\mathbf{u}_{0}$ & No-slip or injection velocity \\
$\Gamma_{2}$ & $\mathbf{u} \cdot \mathbf{t}=\mathbf{a} \cdot \mathbf{t}, p=p_{0}$ & Pressure condition at tube exit with \\
$\Gamma_{3}$ & $\mathbf{u} \cdot \mathbf{n}=\mathbf{b} \cdot \mathbf{n}, \quad \omega=\omega_{0}$ & an unknown velocity distribution \\
\hline
\end{tabular}

Since a Dirichlet boundary condition for the vorticity is imposed, we formulate the problem in terms of the velocity field $\mathbf{u}$, the vorticity $\omega$ and the pressure $p$. We use a modified pressure $p \simeq \frac{1}{\nu} p$ and a modified body force $\mathbf{f} \simeq \frac{1}{\nu} \mathbf{f}$. We are now 
ready to state the following three-field problem:

$$
\begin{aligned}
& \begin{cases}\operatorname{curl} \omega+\nabla p=\mathbf{f} & \text { in } \Omega, \\
\omega=\operatorname{curl} \mathbf{u} & \text { in } \Omega, \\
\operatorname{div} \mathbf{u}=0 & \text { in } \Omega,\end{cases} \\
& \left\{\begin{array}{lll}
\mathbf{u} \cdot \mathbf{n}=0, & \mathbf{u} \cdot \mathbf{t}=0 & \text { on } \Gamma_{1}, \\
\mathbf{u} \cdot \mathbf{t}=0, & p=p_{0} & \text { on } \Gamma_{2}, \\
\mathbf{u} \cdot \mathbf{n}=0, & \omega=\omega_{0} & \text { on } \Gamma_{3} .
\end{array}\right.
\end{aligned}
$$

The remainder of this paper is organized as follows: In Section 2, we introduce the function spaces and derive the variational formulation corresponding to the above problem. In Section 3 we discretize the problem using finite elements of degree 1. Section 4 is devoted to the analysis of the discrete problem. The convergence analysis is performed and error estimates are established. Finally, in Section 5, numerical simulations are presented to illustrate the performance of the proposed approach.

\section{FUnCtionAL FRAMEWORK AND VARIATIONAL FORMULATION}

First, we introduce the following space [13]:

Definition 1. Let $\Gamma_{a}, \Gamma_{b}$ be a partition of the boundary $\Gamma=\partial \Omega$, i.e., $\Gamma_{a} \cap \Gamma_{b}=\emptyset$ and $\bar{\Gamma}_{a} \cup \bar{\Gamma}_{b}=\Gamma$. Let $\gamma_{0}: H^{1}(\Omega) \rightarrow H^{1 / 2}(\Gamma)$ be the trace operator. We define $H_{00}^{1 / 2}\left(\Gamma_{a}\right)$, the set of traces on $\Gamma$ that are equal to 0 on $\Gamma_{b}$, i.e.,

$$
H_{00}^{1 / 2}\left(\Gamma_{a}\right)=\left\{\gamma_{0} \varphi, \varphi \in H^{1}(\Omega) ; \quad \gamma_{0} \varphi=0 \quad \text { on } \quad \Gamma_{b}\right\} .
$$

We denote by $H_{00}^{-1 / 2}\left(\Gamma_{a}\right)$ the dual space of $H_{00}^{1 / 2}\left(\Gamma_{a}\right)$.

We denote by $\partial_{\mathbf{n}}=\mathbf{n} \cdot \nabla$ the normal derivative and by $\partial_{\mathbf{t}}=\mathbf{t} \cdot \nabla$ the tangential derivative along the boundary $\Gamma$.

We consider the following Hilbert spaces:

$$
\begin{array}{lll}
\mathbb{L}^{2}(\Omega)=L^{2}(\Omega) \times L^{2}(\Omega), & & \\
\mathbb{X}=L^{2}(\Omega) \times L_{0}^{2}(\Omega) & \text { when }\left|\Gamma_{2}\right|=0, \\
\mathbb{X}=\mathbb{L}^{2}(\Omega) & \text { when }\left|\Gamma_{2}\right|>0,
\end{array}
$$

where $L_{0}^{2}(\Omega)=\left\{q \in L^{2}(\Omega) ; \int_{\Omega} q d \Omega=0\right\}$. We denote by $\|\cdot\|_{0, \Omega}$ the $L^{2}$-norm in $\Omega$ and endow $\mathbb{X}$ with the following norm:

$$
\|\tau\|_{\mathbb{X}}=\left(\|\theta\|_{0, \Omega}^{2}+\|q\|_{0, \Omega}^{2}\right)^{1 / 2}
$$

for $\tau=(\theta, q) \in \mathbb{X}$. We also consider the Hilbert space $\mathbb{H}(\operatorname{div}, \operatorname{curl} ; \Omega)$ of square integrable vector fields on $\Omega$ whose divergence and rotation are also square integrable:

$$
\mathbb{H}(\operatorname{div}, \operatorname{curl} ; \Omega)=\left\{\mathbf{v} \in \mathbb{L}^{2}(\Omega) ; \operatorname{div} \mathbf{v} \in L^{2}(\Omega), \operatorname{curl} \mathbf{v} \in L^{2}(\Omega)\right\}
$$

Let $\mathbb{M}$ be the closed subspace of $\mathbb{H}(\operatorname{div}$, curl; $\Omega)$ defined by

$$
\mathbb{M}=\left\{\mathbf{v} \in \mathbb{H}(\operatorname{div}, \operatorname{curl} ; \Omega) ;\left.\quad \mathbf{v} \cdot \mathbf{n}\right|_{\Gamma_{1} \cup \Gamma_{3}}=\left.\mathbf{v} \cdot \mathbf{t}\right|_{\Gamma_{1} \cup \Gamma_{2}}=0\right\} .
$$

The boundary condition $\left.\mathbf{v} \cdot \mathbf{n}\right|_{\Gamma_{1} \cup \Gamma_{3}}=0$ is to be understood in the weak sense, i.e.,

$$
\mathbf{v} \cdot \mathbf{n} \in H^{-\frac{1}{2}}(\Gamma) \quad \text { and } \quad\langle\mathbf{v} \cdot \mathbf{n}, \mu\rangle=0 \quad \forall \mu \in H_{00}^{\frac{1}{2}}\left(\Gamma_{1} \cup \Gamma_{3}\right) .
$$

A similar weak sense is given for the boundary condition $\left.\mathbf{v} \cdot \mathbf{t}\right|_{\Gamma_{1} \cup \Gamma_{2}}=0$. 
The spaces $\mathbb{H}(\operatorname{div}, \operatorname{curl} ; \Omega)$ and $\mathbb{M}$ are both equipped with the norm

$$
\|\mathbf{v}\|_{\mathbb{M}}=\left(\|\mathbf{v}\|_{0, \Omega}^{2}+\|\operatorname{div} \mathbf{v}\|_{0, \Omega}^{2}+\|\operatorname{curl} \mathbf{v}\|_{0, \Omega}^{2}\right)^{1 / 2}
$$

and we also consider the semi-norm

$$
|\mathbf{v}|_{\mathbb{M}}=\left(\|\operatorname{div} \mathbf{v}\|_{0, \Omega}^{2}+\|\operatorname{curl} \mathbf{v}\|_{0, \Omega}^{2}\right)^{1 / 2} .
$$

The following results will be useful:

Lemma 1. There exists $s \in] 1 / 2,1]$ such that $\mathbb{M}$ is continuously imbedded in $\mathbb{H}^{s}(\Omega)$.

Proof. We have $\mathbb{M} \subset \mathbb{M}_{1}$, where

$$
\mathbb{M}_{1}=\left\{\mathbf{v} \in \mathbb{H}(\operatorname{div}, \operatorname{curl} ; \Omega) ; \quad \mathbf{v} \cdot \mathbf{n}=0 \text { on } \Gamma_{1} \cup \Gamma_{3}, \quad \mathbf{v} \cdot \mathbf{t}=0 \text { on } \Gamma_{2}\right\} .
$$

For $\mathbf{v} \in \mathbb{M}_{1}$ fixed, let $\varphi$ be the solution of the problem

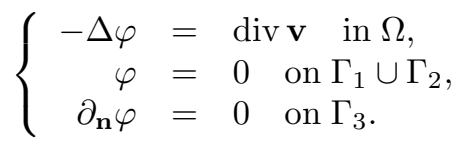

For each of the nonconvex corners $\mathbf{a}_{j}$ of $\Omega$, we can introduce a fixed neighborhood $U_{j}$ (as small as needed) of $\mathbf{a}_{j}$ such that $U_{j} \cap U_{j^{\prime}}=\emptyset$ for each $j \neq j^{\prime}$, with $j, j^{\prime}=$ $1, \ldots, l$. Moreover, as we do not have any of the nonconvex corners in $\left(\overline{\Gamma_{2} \cup \Gamma_{1}}\right) \cap \bar{\Gamma}_{3}$, then, following [11, the solution $\varphi$ can be written as the sum of a regular part $\varphi_{r} \in H^{2}(\Omega)$ and a linear combination $\sum_{j=1}^{k} \lambda_{j} S_{j}$, where the $\lambda_{j}$ are real constants, $S_{j} \in H^{1+s_{j}}(\Omega)$ with compact support in $U_{j}$, and the $s_{j}$ are real numbers such that $1 / 2<s_{j}<\pi / \alpha_{j}$. Therefore, we find that $\varphi \in H^{1+s_{\mathrm{div}}}(\Omega)$ for some positive number $\left.\left.s_{\text {div }} \in\right] 1 / 2,1\right]$. In addition, there is a constant $C$ such that

$$
\|\varphi\|_{1+s_{\mathrm{div}, \Omega}} \leq C\|\operatorname{div} \mathbf{v}\|_{0, \Omega}
$$

Next, we set $\mathbf{w}=\mathbf{v}+\nabla \varphi$. Since $\operatorname{div} \mathbf{w}=0$, there exists a function $\xi \in H^{1}(\Omega)$ such that $\mathbf{w}=\operatorname{curl} \xi$. Moreover, using the fact that $\varphi$ is constant on $\Gamma_{1} \cup \Gamma_{2}$ and assuming now $\mathbf{v} \in \mathbb{M}$, we have

$$
\operatorname{curl} \xi \cdot \mathbf{t}=\partial_{\mathbf{n}} \xi=\mathbf{v} \cdot \mathbf{t}+\nabla \varphi \cdot \mathbf{t}=0, \quad \text { on } \Gamma_{1} \cup \Gamma_{2} .
$$

Then, $\xi$ satisfies the following problem for the Laplace operator:

$$
\left\{\begin{aligned}
-\Delta \xi & =\operatorname{curl} \mathbf{v} \text { in } \Omega, \\
\partial_{\mathbf{n}} \xi & =0 \text { on } \Gamma_{1} \cup \Gamma_{2}, \\
\partial_{\mathbf{t}} \xi & =0 \text { on } \Gamma_{3} .
\end{aligned}\right.
$$

Again, using the regularity results for the Laplacian operator, we find that $\xi \in$ $H^{1+s_{\text {rot }}}(\Omega)$ for some positive number $\left.\left.s_{\text {rot }} \in\right] 1 / 2,1\right]$, and

$$
\|\xi\|_{1+s_{\mathrm{rot}}, \Omega} \leq C\|\operatorname{curl} \mathbf{v}\|_{0, \Omega}
$$

Hence, we obtain

$$
\mathbf{v}=-\nabla \varphi+\operatorname{curl} \xi
$$

and then $\mathbf{v} \in \mathbb{H}^{s}(\Omega)$ for $\left.\left.s=\min \left\{s_{\text {div }}, s_{\text {rot }}\right\} \in\right] 1 / 2,1\right]$ with

$$
\|\mathbf{v}\|_{s, \Omega} \leq\|\nabla \varphi\|_{s, \Omega}+\|\operatorname{curl} \xi\|_{s, \Omega} \leq C|\mathbf{v}|_{\mathbb{M}} \leq C\|\mathbf{v}\|_{\mathbb{M}} .
$$

Corollary 2. Any $\mathbf{v} \in \mathbb{M}$ satisfies $\mathbf{v} \cdot \mathbf{n} \in L^{2}(\Gamma)$ and $\mathbf{v} \cdot \mathbf{t} \in L^{2}(\Gamma)$. 
Hypothesis. We assume throughout this paper that the set

$$
\mathbb{K}=\{\mathbf{v} \in \mathbb{M} ; \quad \operatorname{div} \mathbf{v}=\operatorname{curl} \mathbf{v}=0 \text { a.e. in } \Omega\}
$$

satisfies

$$
\mathbb{K}=\{\mathbf{0}\} .
$$

Remark 1. If $\mathbf{v} \in \mathbb{K}$, then there is a function $\psi \in H^{1}(\Omega) / \mathbb{R}$ such that $\mathbf{v}=\operatorname{curl} \psi$ and $\Delta \psi=0$ a.e. in $\Omega$. Furthermore, $\partial_{\mathbf{n}} \psi=\partial_{\mathbf{t}} \psi=0$ on $\Gamma_{1}, \partial_{\mathbf{t}} \psi=0$ on $\Gamma_{3}$ and $\partial_{\mathbf{n}} \psi=0$ on $\Gamma_{2}$. If $\left|\Gamma_{1}\right|>0$, then Holmgrem's uniqueness theorem insures that $\psi=0$, and then $\mathbf{v}=\mathbf{0}$. If $\left|\Gamma_{1}\right|=0$ and $\Gamma_{3}$ has only one connected component, we have also $\psi=0$, and then $\mathbf{v}=\mathbf{0}$. In these two cases, assumption (2.4) is verified. In the other cases, i.e., when $\left|\Gamma_{1}\right|=0$ and $\Gamma_{3}$ has $m+1$ components with $m \geq 1$, the set $\mathbb{K}$ has a finite dimension equal to $m$ and we can characterize a basis of $\mathbb{K}$. We can work in this framework but with the space $\mathbb{M} / \mathbb{K}$.

Lemma 3. Under assumption (2.4), the semi-norm $|\cdot|_{\mathbb{M}}$ is equivalent to the norm $\|\cdot\|_{\mathbb{M}}$ in $\mathbb{M}$.

Proof. The proof uses a compactness argument. We suppose that the semi-norm $|\cdot|_{\mathbb{M}}$ is not equivalent to the norm $\|\cdot\|_{\mathbb{M}}$ in $\mathbb{M}$. Then, for each integer $n \in \mathbb{N}^{*}$ there exists a sequence $\left(\mathbf{v}_{n}\right)_{n}$ of elements of $\mathbb{M}$ such that

$$
\left\|\mathbf{v}_{n}\right\|_{0, \Omega}=1 \quad \text { and } \quad\left|\mathbf{v}_{n}\right|_{\mathbb{M}}<\frac{1}{n} .
$$

We then have $\left\|\mathbf{v}_{n}\right\|_{\mathbb{M}}<2$. Using Lemma [1, we deduce that the sequence $\left(\mathbf{v}_{n}\right)_{n}$ is bounded in $\mathbb{H}^{s}(\Omega)$ with $\left.\left.s \in\right] 1 / 2,1\right]$. Therefore, there exists a subsequence of $\left(\mathbf{v}_{n}\right)_{n}$, still denoted the same, weakly convergent in $\mathbb{H}^{s}(\Omega)$ and strongly convergent in $\mathbb{L}^{2}(\Omega)$ to some $\mathbf{v}$. Moreover, $\mathbf{v} \in \mathbb{M}$ and $\operatorname{div} \mathbf{v}=\operatorname{curl} \mathbf{v}=0$ a.e. in $\Omega$. From hypothesis (2.4), we deduce that $\mathbf{v}=\mathbf{0}$. On the other hand, as the sequence converges strongly in $\mathbb{L}^{2}(\Omega)$ to $\mathbf{v}$, then $\|\mathbf{v}\|_{0, \Omega}=1$. The latter result contradicts $\mathbf{v}=\mathbf{0}$.

In order to define the trace of the elements of the space $H=\left\{\theta \in L^{2}(\Omega) ; \Delta \theta \in\right.$ $\left.H^{-1}(\Omega)\right\}$, we prove the following lemma.

Lemma 4. Set $Q=H_{0}^{1}(\Omega) \cap H^{2}(\Omega)$ and consider the space $Y$ defined by

$$
Y=\left\{\mu \in L^{2}(\Gamma) ; \exists \varphi \in Q, \mu=\partial_{\mathbf{n}} \varphi \text { a.e. in } \Gamma\right\} \text {. }
$$

For all functions $\theta \in H$, the trace $\gamma_{0}(\theta)$ is defined on the dual space $Y^{\prime}$.

Proof. The space $Y$ is normed by

$$
\|\mu\|_{Y}=\operatorname{Inf}_{\varphi \in Q, \partial_{\mathbf{n}} \varphi=\mu}|\varphi|_{2, \Omega} .
$$

Using [11], we can define a continuous linear operator $\gamma_{0}$ from $H$ to $Y^{\prime}$ such that

$$
\forall \theta \in D(\bar{\Omega}), \gamma_{0}(\theta)=\theta_{\mid \Gamma}
$$

and

$$
\forall \theta \in H, \forall \varphi \in Q, \quad \int_{\Omega} \theta \Delta \varphi d x-\langle\Delta \theta, \varphi\rangle_{-1,1, \Omega}=\left\langle\gamma_{0}(\theta), \partial_{\mathbf{n}} \varphi\right\rangle_{Y^{\prime}, Y}
$$


Throughout this section, we will denote by $(\cdot, \cdot)$ the scalar product in $L^{2}(\Omega)$, by $\langle\cdot, \cdot\rangle$ the duality in the space $\mathbb{M}$ and by $\langle\cdot, \cdot\rangle_{\Gamma_{i}}$ the scalar product in $L^{2}\left(\Gamma_{i}\right)$ for $i=2,3$. We assume that $\mathbf{f}$ belongs to $\mathbb{L}^{2}(\Omega)$ and, for the sake of simplicity, we take $p_{0} \in L^{2}(\Gamma)$ and $\omega_{0} \in L^{2}(\Gamma)$. We can take, of course, weaker conditions for $p_{0}$ and $\omega_{0}$. Using integration by parts, one can derive the following variational formulation for problem (1.1)-(1.2):

$$
\left\{\begin{array}{rll}
\text { Find } \sigma=(\omega, p) \in \mathbb{X} \text { and } \mathbf{u} \in \mathbb{M} \text { such that } \\
(\omega, \operatorname{curl} \mathbf{v})-(p, \operatorname{div} \mathbf{v}) & =F(\mathbf{v}), & \forall \mathbf{v} \in \mathbb{M}, \\
(\omega, \theta)-(\theta, \operatorname{curl} \mathbf{u}) & =0, & \forall \theta \in L^{2}(\Omega), \\
(q, \operatorname{div} \mathbf{u}) & =0, & \forall q \in L^{2}(\Omega),
\end{array}\right.
$$

where $F \in \mathbb{M}^{\prime}$ is given by

$$
F(\mathbf{v})=(\mathbf{f}, \mathbf{v})+\left\langle\omega_{0}, \mathbf{v} \cdot \mathbf{t}\right\rangle_{\Gamma_{3}}-\left\langle p_{0}, \mathbf{v} \cdot \mathbf{n}\right\rangle_{\Gamma_{2}}, \forall \mathbf{v} \in \mathbb{M} .
$$

By adding the second equation of (2.5) to the third one, we obtain

$$
\begin{aligned}
(\omega, \theta)-(\theta, \operatorname{curl} \mathbf{u})+(q, \operatorname{div} \mathbf{u}) & =0, & \forall \tau=(\theta, q) \in \mathbb{X} \\
(\omega, \operatorname{curl} \mathbf{v})-(p, \operatorname{div} \mathbf{v}) & =F(\mathbf{v}), & \forall \mathbf{v} \in \mathbb{M} .
\end{aligned}
$$

Now we consider the bilinear forms $a: \mathbb{X} \times \mathbb{X} \rightarrow \mathbb{R}$ and $b: \mathbb{X} \times \mathbb{M} \rightarrow \mathbb{R}$ defined for all $\sigma=(\omega, p), \tau=(\theta, q) \in \mathbb{X}$ and $\mathbf{v} \in \mathbb{M}$ by

$$
a(\sigma, \tau)=(\omega, \theta) \quad \text { and } \quad b(\tau, \mathbf{v})=-(\theta, \operatorname{curl} \mathbf{v})+(q, \operatorname{div} \mathbf{v}) .
$$

We then obtain the following saddle point formulation associated to problem (1.1)(1.2):

$$
\left\{\begin{array}{cl}
\text { Find } \quad(\sigma, \mathbf{u}) \in \mathbb{X} \times \mathbb{M} & \text { such that } \\
a(\sigma, \tau)+b(\tau, \mathbf{u})=0, & \forall \tau \in \mathbb{X} \\
b(\sigma, \mathbf{v})=-F(\mathbf{v}), & \forall \mathbf{v} \in \mathbb{M} .
\end{array}\right.
$$

We denote by $\mathbb{V}$ the kernel of $b$, i.e.,

$$
\mathbb{V}=\{\tau \in \mathbb{X} ; b(\tau, \mathbf{v})=0, \quad \forall \mathbf{v} \in \mathbb{M}\}
$$

We remark that if $\tau=(\theta, q) \in \mathbb{V}$, then we have $\operatorname{curl} \theta+\nabla q=0$. So, we deduce that $\Delta \theta=\Delta q=0$ a.e. in $\Omega$. Hence, using Lemma 4, we can define the traces of $\theta$ and $q$ on the boundary $\Gamma$ as elements of $Y^{\prime}$. We take $\mu \in Y$ with $\mu=0$ on $\Gamma_{1} \cup \Gamma_{2}$ and consider a function $\varphi \in Q$ such that $\partial_{n} \varphi=\mu$ on $\Gamma$. Then, for $\mathbf{v}=\operatorname{curl} \varphi$, we have $\mathbf{v} \cdot \mathbf{n}=0$ a.e. on $\Gamma, \mathbf{v} \cdot \mathbf{t}=-\mu$ a.e. on $\Gamma, \mathbf{v} \in \mathbb{M}$ and $\operatorname{div} \mathbf{v}=0$. From the definition of $\mathbb{V}$, we deduce that

$$
\int_{\Omega} \theta \operatorname{curl} \mathbf{v} d x=-\int_{\Omega} \theta \Delta \varphi d x=-\left\langle\gamma_{0}(\theta), \mu\right\rangle=0 .
$$

Since $\mu=0$ on $\Gamma_{1} \cup \Gamma_{2}$, the previous equality can be written as follows:

$$
\gamma_{0}(\theta)=0 \text { on } \Gamma_{3} \text { in the sense of } Y^{\prime} .
$$

Similarly, we establish that $\gamma_{0}(q)=0$ on $\Gamma_{2}$ (in the sense of $Y^{\prime}$ ). Then, we have the following characterization:

$$
\mathbb{V}=\left\{\tau=(\theta, q) \in \mathbb{X} ; \operatorname{curl} \theta+\nabla q=\mathbf{0} \text { a.e. in } \Omega, " q=0 \text { on } \Gamma_{2} ", " \theta=0 \text { on } \Gamma_{3} "\right\} \text {. }
$$

Therefore, when $\tau=(\theta, q) \in \mathbb{V}$, then $q \in Z$, where $Z$ is given by

$$
\begin{array}{ll}
Z=\left\{q \in L_{0}^{2}(\Omega) ; \Delta q=0 \text { a.e. in } \Omega\right\} & \text { if }\left|\Gamma_{2}\right|=0, \\
Z=\left\{q \in L^{2}(\Omega) ; \Delta q=0 \text { a.e. in } \Omega, q=0 \text { on } \Gamma_{2}\right\} & \text { if } \quad\left|\Gamma_{2}\right|>0 .
\end{array}
$$


We recall that there exists a positive constant $C$, depending only on $\Omega$, such that,

$$
\|q\|_{0, \Omega} \leq C\|\nabla q\|_{-1, \Omega}, \quad \forall q \in L_{0}^{2}(\Omega)
$$

where $\|\cdot\|_{-1, \Omega}$ denotes the norm of $H^{-1}(\Omega)$, the dual space of $H_{0}^{1}(\Omega)$. When $\left|\Gamma_{2}\right|=$ 0 , the inequality (2.8) is true for every $q \in Z$. This result remains valid when $\left|\Gamma_{2}\right|>0$. This property is stated by the following lemma:

Lemma 5. There is a positive constant $C$, depending only on $\Omega$, such that

$$
\|q\|_{0, \Omega} \leq C\|\nabla q\|_{-1, \Omega}, \quad \forall q \in Z .
$$

Proof. Suppose that, when $\left|\Gamma_{2}\right|>0$, (2.9) does not hold. Then, there is a sequence $\left(q_{n}\right)_{n}$ of elements of $Z$ such that

$$
\left\|q_{n}\right\|_{0, \Omega}>n\left\|\nabla q_{n}\right\|_{-1, \Omega}
$$

for all $n \in \mathbb{N}$. Let $\bar{q}_{n}=\frac{1}{\left\|q_{n}\right\|_{0, \Omega}} q_{n}$; then $\left\|\bar{q}_{n}\right\|_{0, \Omega}=1$ and $\frac{1}{n}>\left\|\nabla \bar{q}_{n}\right\|_{-1, \Omega}$.

This inequality implies that $\left(\nabla \bar{q}_{n}\right)_{n}$ goes to 0 in $\mathbb{H}^{-1}(\Omega)$. Hence, there exists a subsequence of $\left(\bar{q}_{n}\right)_{n}$, still denoted the same, weakly convergent in $L^{2}(\Omega)$ to some $\bar{q} \in Z$. We introduce $\tilde{q}_{n} \in L_{0}^{2}(\Omega)$ by $\tilde{q}_{n}=\bar{q}_{n}-\frac{1}{|\Omega|} \int_{\Omega} \bar{q}_{n} d \Omega$. This sequence converges weakly in $L^{2}(\Omega)$ to $\tilde{q} \in L_{0}^{2}(\Omega)$ given by $\tilde{q}=\bar{q}-\frac{1}{|\Omega|} \int_{\Omega} \bar{q} d \Omega$. Moreover, there is a constant $C>0$ such that

$$
\left\|\tilde{q}_{n}\right\|_{0, \Omega} \leq C\left\|\nabla \tilde{q}_{n}\right\|_{-1, \Omega}=C\left\|\nabla \bar{q}_{n}\right\|_{-1, \Omega} \leq \frac{C}{n} .
$$

Therefore, $\left(\tilde{q}_{n}\right)_{n}$ tends strongly to 0 in $L^{2}(\Omega)$. Moreover, since $\left(\bar{q}_{n}\right)_{n}$ converges weakly to $\bar{q}$ in $L^{2}(\Omega)$, we obtain that $\left(\bar{q}_{n}\right)_{n}$ tends to $\bar{q}=\frac{1}{|\Omega|} \int_{\Omega} \bar{q} d \Omega$ strongly in $L^{2}(\Omega)$. Finally, using the fact that $\bar{q} \in Z$ and $\left|\Gamma_{2}\right|>0$, we have necessarily $\bar{q}=0$. On the other hand, we have $\left\|\bar{q}_{n}\right\|_{0, \Omega}=1$ for every $n$. Then $\|\bar{q}\|_{0, \Omega}=1$, which contradicts $\bar{q}=0$.

Corollary 6. We have

$$
\|\tau\|_{\mathbb{X}} \leq\left(1+C^{2}\right)^{\frac{1}{2}}\|\theta\|_{0, \Omega}, \quad \forall \tau=(\theta, q) \in \mathbb{V},
$$

where the constant $C$ is given by Lemma 5 .

Proof. For all $\tau=(\theta, q) \in \mathbb{V}$ we have $\operatorname{curl} \theta+\nabla q=0$. Therefore, it follows from Lemma 5 that

$$
\|q\|_{0, \Omega} \leq C\|\nabla q\|_{-1, \Omega}=C\|\operatorname{curl} \theta\|_{-1, \Omega} \leq C\|\theta\|_{0, \Omega} .
$$

Hence,

$$
\|\tau\|_{\mathbb{X}}^{2}=\|\theta\|_{0, \Omega}^{2}+\|q\|_{0, \Omega}^{2} \leq\left(1+C^{2}\right)\|\theta\|_{0, \Omega}^{2}
$$

Theorem 7. Let $\mathbf{f} \in \mathbb{L}^{2}(\Omega), p_{0} \in L^{2}(\Gamma)$ and $\omega_{0} \in L^{2}(\Gamma)$. Then, the saddle point problem (2.7) admits a unique solution $\sigma=(\omega, p) \in \mathbb{X}$ and $\mathbf{u} \in \mathbb{M}$ satisfying

$$
\begin{cases}\operatorname{curl} \omega+\nabla p=\mathbf{f} & \text { in } \mathbb{L}^{2}(\Omega), \\ \omega=\operatorname{curl} \mathbf{u} & \text { in } L^{2}(\Omega), \\ \operatorname{div} \mathbf{u}=0 & \text { in } L^{2}(\Omega),\end{cases}
$$


and

$$
\left\{\begin{array}{llll}
\mathbf{u} \cdot \mathbf{n}=0 & \text { a.e. on } \Gamma_{1}, & \mathbf{u} \cdot \mathbf{t}=0 & \text { a.e. on } \Gamma_{1}, \\
p=p_{0} & \text { a.e. on } \Gamma_{2}, & \mathbf{u} \cdot \mathbf{t}=0 & \text { a.e. on } \Gamma_{2}, \\
\mathbf{u} \cdot \mathbf{n}=0 & \text { a.e. on } \Gamma_{3}, & \omega=\omega_{0} & \text { a.e. on } \Gamma_{3} .
\end{array}\right.
$$

Proof. First, one can easily verify that the forms $a$ and $b$ (see (2.6) ) are bilinear and continuous. Second, the existence and uniqueness of the solution of problem (2.7) is then established once we prove that $a$ is $\mathbb{V}$-elliptic and $b$ satisfies the "inf-sup" condition [10]. The coercivity of $a$ on $\mathbb{V}$ is a consequence of Corollary 6 , Next, we check the "inf-sup" condition on $b$. For a given $\mathbf{v} \in \mathbb{M}$, we set $\bar{\tau}=(-\operatorname{curl} \mathbf{v}, \operatorname{div} \mathbf{v}) \in$ $\mathbb{X}$. Then,

$$
b(\bar{\tau}, \mathbf{v})=\|\bar{\tau}\|_{\mathbb{X}}^{2}=\|\operatorname{curl} \mathbf{v}\|_{0, \Omega}^{2}+\|\operatorname{div} \mathbf{v}\|_{0, \Omega}^{2}
$$

and

$$
\sup _{\tau \in \mathbb{X}} \frac{b(\tau, \mathbf{v})}{\|\tau\|_{\mathbb{X}}} \geq \frac{b(\bar{\tau}, \mathbf{v})}{\|\bar{\tau}\|_{\mathbb{X}}}=\|\bar{\tau}\|_{\mathbb{X}}=|\mathbf{v}|_{\mathbb{M}}
$$

Hence, the "inf-sup" condition is satisfied. Therefore, we conclude the existence and uniqueness of a pair $(\sigma, \mathbf{u}) \in \mathbb{X} \times \mathbb{M}$, with $\sigma=(\omega, p)$, that is a solution of (2.7). In addition, one can easily verify that $(\omega, p, \mathbf{u})$ satisfies (2.11) by simply using in (2.7) $\tau=(\theta, \eta) \in \mathcal{D}^{2}(\Omega)$ and $\mathbf{v} \in \mathcal{D}^{2}(\Omega)$. The normal and tangential boundary conditions for the solution $\mathbf{u}$ are satisfied because $\mathbf{u} \in \mathbb{M}$. We only have to check the boundary data for the pressure on $\Gamma_{2}$ and vorticity on $\Gamma_{3}$. Since $\mathbf{f} \in L^{2}(\Omega)$, by applying the differential operators curl and div to the first equation of (2.11), we obtain that both $\Delta \omega$ and $\Delta p$ belong to $H^{-1}(\Omega)$. Therefore, using Lemma 4 the traces of $\omega$ and $p$ are defined in $Y^{\prime}$. Let $\mu$ be an element of $Y$ such that $\mu=0$ on $\Gamma_{1} \cup \Gamma_{2}$. Taking $\varphi$ in $Q$ with $\partial_{n} \varphi=\mu$ on $\Gamma$, the function $\mathbf{v}=\operatorname{curl} \varphi$ satisfies $\mathbf{v} \cdot \mathbf{n}=0$ a.e. on $\Gamma$ and $\mathbf{v} \cdot \mathbf{t}=-\mu$ a.e. on $\Gamma$. Consequently, $\mathbf{v} \in \mathbb{M}$ and $\operatorname{div} \mathbf{v}=0$. Choosing $\mathbf{v}$ as test function in (2.7), we obtain

$$
\int_{\Omega} \omega \operatorname{curl} \mathbf{v} d x=\int_{\Omega} \mathbf{f} \cdot \mathbf{v} d x+\int_{\Gamma_{3}} \omega_{0} \mathbf{v} \cdot \mathbf{t} d \sigma
$$

On the other hand, we also have

$$
\int_{\Omega} \mathbf{f} \cdot \operatorname{curl} \varphi d x=\langle\operatorname{curl} \mathbf{f}, \varphi\rangle_{-1,1, \Omega}
$$

and $\operatorname{curl} \mathbf{f}=-\Delta \omega$. Therefore, we obtain

$$
\left\langle\gamma_{0} \omega, \mu\right\rangle_{Y^{\prime}, Y}=\int_{\Gamma_{3}} \omega_{0} \mu d \sigma
$$

i.e., $\gamma_{0} \omega=\omega_{0}$ on $\Gamma_{3}$ in the sense of $Y^{\prime}$. Similarly, one can also prove that $\gamma_{0} p=p_{0}$ on $\Gamma_{2}$ in the sense of $Y^{\prime}$.

\section{The DisCRETE PROBLEM}

Let $\left(\mathcal{T}_{h}\right)_{h}$ be a regular family of triangulations of $\bar{\Omega}$. For each triangle $K$, we denote by $h_{K}$ its diameter, and by $|K|$ its area. We associate to each triangulation $\mathcal{T}_{h}$ the following sets:

- $\mathcal{E}_{h}$ is the set of the internal edges.

- $\mathcal{F}_{h}^{i}$ is the set of the edges which belong to the part $\Gamma_{i}$ of the boundary $(i=1,2,3)$.

- $\mathcal{C}_{h}=\mathcal{E}_{h} \cup \mathcal{F}_{h}^{1} \cup \mathcal{F}_{h}^{2} \cup \mathcal{F}_{h}^{3}$. $\mathcal{C}_{h}$ is the set of all the edges of $\mathcal{T}_{h}$. 
We assume that if an edge $e$ belongs to $\Gamma$, then it belongs entirely to one of the $\mathcal{F}_{h}^{i}$, i.e., $e \subset \Gamma_{i}$. For each edge $e \in \mathcal{E}_{h}$, there exist two triangles $K$ and $K^{\prime}$ in $\mathcal{T}_{h}$ such that $e=\partial K \cap \partial K^{\prime}$. We denote by $h_{e}$ the length of each edge $e$ and we set $h=\max _{K \in \mathcal{T}_{h}} h_{K}$. Moreover, since $\left(\mathcal{T}_{h}\right)_{h}$ is a regular family of triangulations, there is a constant $\widehat{c}>0$, independent of $h$, such that

$$
\forall h>0, \quad \forall K \in \mathcal{T}_{h}, \quad \forall e \subset \partial K \quad h_{K} \leq \hat{c} h_{e} .
$$

For every $l \in \mathbb{N}$ and $K \in \mathcal{T}_{h}$ we denote by $P_{l}(K)$ the space of the polynomial functions defined on $K$ of degree less than or equal to $l$, and by $\mathbb{P}_{l}(K)$ the space $P_{l}(K) \times P_{l}(K)$. We introduce the following discrete spaces:

$$
\begin{aligned}
L_{h} & =\left\{q_{h} \in L^{2}(\Omega) ; q_{\left.h\right|_{K}} \in P_{0}(K) \forall K \in \mathcal{T}_{h}\right\}, \\
\mathbb{X}_{h} & =L_{h} \times L_{h} \cap \mathbb{X}, \\
\mathbb{M}_{h} & =\left\{\mathbf{v}_{h} \in\left(C^{0}(\bar{\Omega})\right)^{2} ; \mathbf{v}_{\left.h\right|_{K}} \in \mathbb{P}_{1}(K) \forall K \in \mathcal{T}_{h}\right\} \cap \mathbb{M}, \\
& =\left\{\mathbf{v}_{h} \in \mathbb{M} ; \mathbf{v}_{\left.h\right|_{K}} \in \mathbb{P}_{1}(K) \forall K \in \mathcal{T}_{h}\right\} .
\end{aligned}
$$

The discrete formulation for the saddle point problem (2.7) is given by

$$
\begin{cases}\text { Find }\left(\sigma_{h}, \mathbf{u}_{h}\right) \in \mathbb{X}_{h} \times \mathbb{M}_{h} & \text { such that } \\ a\left(\sigma_{h}, \tau_{h}\right)+b\left(\tau_{h}, \mathbf{u}_{h}\right)=0 & \forall \tau_{h} \in \mathbb{X}_{h}, \\ b\left(\sigma_{h}, \mathbf{v}_{h}\right)=-F\left(\mathbf{v}_{h}\right) & \forall \mathbf{v}_{h} \in \mathbb{M}_{h},\end{cases}
$$

with $\sigma_{h}=\left(\omega_{h}, p_{h}\right)$. We recall that the bilinear forms $a$ and $b$ and the linear form $F$ are given by

$$
\begin{aligned}
& a\left(\sigma_{h}, \tau_{h}\right)=\left(\omega_{h}, \theta_{h}\right), \\
& b\left(\tau_{h}, \mathbf{v}_{h}\right)=-\left(\theta_{h}, \operatorname{curl} \mathbf{v}_{h}\right)+\left(q_{h}, \operatorname{div} \mathbf{v}_{h}\right), \\
& F\left(\mathbf{v}_{h}\right)=\left(\mathbf{f}, \mathbf{v}_{h}\right)+\left\langle\omega_{0}, \mathbf{v}_{h} \cdot \mathbf{t}\right\rangle_{\Gamma_{3}}-\left\langle p_{0}, \mathbf{v}_{h} \cdot \mathbf{n}\right\rangle_{\Gamma_{2}},
\end{aligned}
$$

where $\sigma_{h}=\left(\omega_{h}, p_{h}\right) \in \mathbb{X}_{h}, \tau_{h}=\left(\theta_{h}, q_{h}\right) \in \mathbb{X}_{h}$ and $\mathbf{v}_{h} \in \mathbb{M}_{h}$.

The choice of the spaces $\mathbb{X}_{h}$ and $\mathbb{M}_{h}$ allows the bilinear form $b$ to inherit the inf-sup condition satisfied in the continuous case. Indeed, for $\mathbf{v}_{h} \in \mathbb{M}_{h}$ and $\bar{\tau}_{h}=$ $\left(-\operatorname{curl} \mathbf{v}_{h}, \operatorname{div} \mathbf{v}_{h}\right) \in \mathbb{X}_{h}$, we have

$$
b\left(\bar{\tau}_{h}, \mathbf{v}_{h}\right)=\left\|\bar{\tau}_{h}\right\|_{\mathbb{X}}^{2}=\left\|\operatorname{curl} \mathbf{v}_{h}\right\|_{0, \Omega}^{2}+\left\|\operatorname{div} \mathbf{v}_{h}\right\|_{0, \Omega}^{2}=\left|\mathbf{v}_{h}\right|_{\mathbb{M}}^{2} .
$$

Hence,

$$
\sup _{\tau_{h} \in \mathbb{X}_{h}} \frac{b\left(\tau_{h}, \mathbf{v}_{h}\right)}{\left\|\tau_{h}\right\|_{\mathbb{X}}} \geq \frac{b\left(\bar{\tau}_{h}, \mathbf{v}_{h}\right)}{\left\|\bar{\tau}_{h}\right\|_{\mathbb{X}}}=\left\|\bar{\tau}_{h}\right\|_{\mathbb{X}}=\left|\mathbf{v}_{h}\right|_{\mathbb{M}} .
$$

This shows that the discrete "inf-sup" condition holds.

In order to follow the standard analysis (see for example [10]), we need to obtain the coercivity of $a$ on the discrete kernel

$$
\mathbb{V}_{h}=\left\{\tau_{h} \in \mathbb{X}_{h} ; b\left(\tau_{h}, \mathbf{v}_{h}\right)=0 \forall \mathbf{v}_{h} \in \mathbb{M}_{h}\right\} .
$$

It is clear that we do not have $\mathbb{V}_{h} \subset \mathbb{V}$. Hence, the coercivity of the form $a$ on $\mathbb{V}_{h}$ is not a consequence of Corollary 6. In fact, one can prove that $a$ is not coercive on $\mathbb{V}_{h}$. Indeed, analyzing the uniqueness of the solution of problem (3.3) leads to a solution for $F=0$. In this case, we have $b\left(\sigma_{h}, \mathbf{u}_{h}\right)=0$. Thus, $a\left(\sigma_{h}, \sigma_{h}\right)=0$. Due to the expression of $a$, it follows that $a\left(\sigma_{h}, \tau_{h}\right)=0, \forall \tau_{h} \in \mathbb{X}_{h}$. Hence, using the inf-sup condition, we deduce that $\mathbf{u}_{h}=0$. Moreover, we have $\left(p_{h}, \operatorname{div} \mathbf{v}_{h}\right)=0, \forall \mathbf{v}_{h} \in \mathbb{M}_{h}$. Unfortunately, this does not imply that $p_{h}=0$. Therefore, the homogeneous problem (3.3) admits nontrivial solutions, and so $a$ is not coercive on $\mathbb{V}_{h}$. To restore the coercivity, one needs to modify the bilinear form 
and not the spaces in order to preserve the uniform "inf-sup" condition satisfied by the bilinear form $b$. To do this, we adopt the approach developed in [1] and [2] and tailor it to our problem. First, we observe that the proof of Lemma 5 leans essentially on the fact that $\operatorname{curl} \theta+\nabla q=\mathbf{0}$. This crucial property is no longer valid at the discrete level. One can only estimate the norm $\|\operatorname{curl} \theta+\nabla q\|_{-1, \Omega}$ in terms of the jumps across the edges of the elements. However, we will see that this property is enough for our objective.

For a given edge $e \in \mathcal{E}_{h}$, we have $e=\partial K \cap \partial K^{\prime}$ for some $K \neq K^{\prime} \in \mathcal{T}_{h}$. Let $\mathbf{n}_{e}^{K}$ and $\mathbf{t}_{e}^{K}$ be the outward normal and tangent vectors to the edge $e$ with respect to the triangle $K$. Then $\mathbf{n}_{e}^{K}+\mathbf{n}_{e}^{K^{\prime}}=\mathbf{t}_{e}^{K}+\mathbf{t}_{e}^{K^{\prime}}=\mathbf{0}$ on $e$. Also, for any edge $e \in \mathcal{F}_{h}^{i}$ with $i=1,2,3$, we have $e \subset \partial K$ for some $K \in \mathcal{T}_{h}$. To simplify the notation, we let $\mathbf{n}_{e}=\mathbf{n}_{e}^{K}$ and $\mathbf{t}_{e}=\mathbf{t}_{e}^{K}$ be the outward normal and tangent vectors to the edge $e$ with respect to the triangle $K$.

Definition 2. For $\tau_{h}=\left(\theta_{h}, q_{h}\right) \in \mathbb{X}_{h}$, we define the jump $\left[\tau_{h}\right]_{e}$ across an edge $e$ of $\mathcal{T}_{h}$ as follows:

- $e \in \mathcal{E}_{h}$ and $e=\partial K \cap \partial K^{\prime}$

- $e \in \mathcal{F}_{h}^{1}$ and $e \subset \partial K$

- $e \in \mathcal{F}_{h}^{2}$ and $e \subset \partial K$,

- $e \in \mathcal{F}_{h}^{3}$ and $e \subset \partial K$,

$$
\begin{aligned}
& {\left[\tau_{h}\right]_{e}=\left(\theta_{h}^{K}-\theta_{h}^{K^{\prime}}\right) \mathbf{t}_{e}^{K}-\left(q_{h}^{K}-q_{h}^{K^{\prime}}\right) \mathbf{n}_{e}^{K}} \\
& {\left[\tau_{h}\right]_{e}=0} \\
& {\left[\tau_{h}\right]_{e}=-q_{h}^{K} \mathbf{n}_{e}} \\
& {\left[\tau_{h}\right]_{e}=\theta^{K} \mathbf{t}_{e} .}
\end{aligned}
$$

We also define the jumps $\left[\theta_{h}\right]_{e}$ and $\left[q_{h}\right]_{e}$ across any edge $e$ of $\mathcal{T}_{h}$ as follows:
- $e \in \mathcal{E}_{h}$ and $e=\partial K \cap \partial K^{\prime}$
- $e \in \mathcal{F}_{h}^{1}$ and $e \subset \partial K$
$\left[\theta_{h}\right]_{e}=\left(\theta_{h}^{K}-\theta_{h}^{K^{\prime}}\right)$,
$\left[\theta_{h}\right]_{e}=0$
- $e \in \mathcal{F}_{h}^{2}$ and $e \subset \partial K$,
$\left[\theta_{h}\right]_{e}=0$
$\left[\theta_{h}\right]_{e}=\theta^{K}$,
$\left[q_{h}\right]_{e}=\left(q_{h}^{K^{\prime}}-q_{h}^{K}\right)$,
$\left[q_{h}\right]_{e}=0$,
$\left[q_{h}\right]_{e}=-q_{h}^{K}$,
$\left[q_{h}\right]_{e}=0$,

For every $e \in \mathcal{C}_{h}$, we have

$$
\left[\tau_{h}\right]_{e}=\left[\theta_{h}\right]_{e} \mathbf{t}_{e}^{K}+\left[q_{h}\right]_{e} \mathbf{n}_{e}^{K} .
$$

We consider the symmetric bilinear form $A_{h}: \mathbb{X}_{h} \times \mathbb{X}_{h} \rightarrow \mathbb{R}$ given by

$$
A_{h}\left(\delta_{h}, \tau_{h}\right)=\sum_{e \in \mathcal{C}_{h}} h_{e}\left(\left[\delta_{h}\right]_{e},\left[\tau_{h}\right]_{e}\right)_{e}, \quad \forall \delta_{h}, \tau_{h} \in \mathbb{X}_{h}
$$

and the associated semi-norm on $\mathbb{X}_{h}$ defined by

$$
\left|\tau_{h}\right|_{h}=\sqrt{A_{h}\left(\tau_{h}, \tau_{h}\right)}=\left(\sum_{e \in \mathcal{C}_{h}} h_{e}\left\|\left[\tau_{h}\right]_{e}\right\|_{0, e}^{2}\right)^{\frac{1}{2}}, \quad \forall \tau_{h} \in \mathbb{X}_{h} .
$$

Using the Cauchy-Schwarz inequality, we have

$$
\left|A_{h}\left(\delta_{h}, \tau_{h}\right)\right| \leq\left|\delta_{h}\right|_{h}\left|\tau_{h}\right|_{h}, \quad \forall \delta_{h}, \tau_{h} \in \mathbb{X}_{h} .
$$

Moreover,

$$
A_{h}\left(\tau_{h}, \tau_{h}\right)=\left|\tau_{h}\right|_{h}^{2}, \forall \tau_{h} \in \mathbb{X}_{h}
$$

We also observe that

$\left(\left[\delta_{h}\right]_{e},\left[\tau_{h}\right]_{e}\right)_{e}=\left(\left[\rho_{h}\right]_{e},\left[\theta_{h}\right]_{e}\right)_{e}+\left(\left[r_{h}\right]_{e},\left[q_{h}\right]_{e}\right)_{e}, \quad \forall \delta_{h}=\left(\rho_{h}, r_{h}\right), \tau_{h}=\left(\theta_{h}, q_{h}\right) \in \mathbb{X}_{h}$.

Next, we consider the linear form $G_{h}: \mathbb{X}_{h} \rightarrow \mathbb{R}$ given by

$$
G_{h}\left(\tau_{h}\right)=-\sum_{e \in \mathcal{F}_{h}^{2}} h_{e}\left(p_{0} \mathbf{n}_{e},\left[\tau_{h}\right]_{e}\right)_{e}+\sum_{e \in \mathcal{F}_{h}^{3}} h_{e}\left(\omega_{0} \mathbf{t}_{e},\left[\tau_{h}\right]_{e}\right)_{e}, \quad \forall \tau_{h}=\left(\theta_{h}, q_{h}\right) \in \mathbb{X}_{h},
$$


which also satisfies

$$
G_{h}\left(\tau_{h}\right)=-\sum_{e \in \mathcal{F}_{h}^{2}} h_{e}\left(p_{0},\left[q_{h}\right]_{e}\right)_{e}+\sum_{e \in \mathcal{F}_{h}^{3}} h_{e}\left(\omega_{0},\left[\theta_{h}\right]_{e}\right)_{e}
$$

Remark 2. The jumps are constant on the edges.

For a positive fixed parameter $\beta_{h}>0$, we define the stabilized bilinear form $a_{h}: \mathbb{X}_{h} \times \mathbb{X}_{h} \rightarrow \mathbb{R}$ given by

$$
a_{h}\left(\delta_{h}, \tau_{h}\right)=a\left(\delta_{h}, \tau_{h}\right)+\beta_{h} A_{h}\left(\delta_{h}, \tau_{h}\right) \quad \forall \delta_{h}, \tau_{h} \in \mathbb{X}_{h} .
$$

The discrete problem (3.3) is then modified as follows:

$$
\left\{\begin{array}{cc}
\text { Find }\left(\sigma_{h}, \mathbf{u}_{h}\right) \in \mathbb{X}_{h} \times \mathbb{M}_{h} \text { with } \quad \sigma_{h}=\left(\omega_{h}, p_{h}\right) \quad \text { such that } \\
a_{h}\left(\sigma_{h}, \tau_{h}\right)+b\left(\tau_{h}, \mathbf{u}_{h}\right)=\beta_{h} G_{h}\left(\tau_{h}\right), & \forall \tau_{h} \in \mathbb{X}_{h}, \\
b\left(\sigma_{h}, \mathbf{v}_{h}\right)=-F\left(\mathbf{v}_{h}\right), & \forall \mathbf{v}_{h} \in \mathbb{M}_{h} .
\end{array}\right.
$$

We note that for $\beta_{h}=0$, problem (3.12) is reduced to problem (3.3). Moreover, we have

Theorem 8. For any $\beta_{h}>0$, problem (3.12) admits a unique solution.

Proof. To establish the uniqueness of the solution, we consider problem (3.12) with $F=0$ and $G_{h}=0$. It follows that $b\left(\sigma_{h}, \mathbf{u}_{h}\right)=0$. Then, $a_{h}\left(\sigma_{h}, \sigma_{h}\right)=0$, which gives $\omega_{h}=0$ and $A_{h}\left(\sigma_{h}, \sigma_{h}\right)=\left|\sigma_{h}\right|_{h}^{2}=0$.

Then for $e \in \mathcal{C}_{h},\left\|\left[\sigma_{h}\right]_{e}\right\|_{0, e}=0$, i.e., for all $e \in \mathcal{E}_{h}\left\|\left[\sigma_{h}\right]_{e}\right\|_{0, e}=\left\|\left[p_{h}\right]_{e}\right\|_{0, e}=0$ and for all $e \in \mathcal{F}_{h}^{2}\left\|\left[\sigma_{h}\right]_{e}\right\|_{0, e}=\left\|p_{h}\right\|_{0, e}=0$. We deduce then that $p_{h}=0$.

We get from $b\left(\tau_{h}, \mathbf{u}_{h}\right)=0 \forall \tau_{h} \in \mathbb{X}_{h}$ and the "inf-sup" condition that $\mathbf{u}_{h}=0$. This proves the uniqueness. Moreover, because of the linearity of problem (3.12), we deduce the existence of the solution.

The bilinear form $A_{h}$ gives the $\mathbb{V}_{h}$ coercivity of the new form $a_{h}$. This coercivity is not a priori uniform, i.e., the constant of ellipticity can depend on $h$. We study this coercivity and also the continuity of $A_{h}$ in the following results. The form $G_{h}$ is introduced to preserve the consistency. We first focus on the coercivity of $a_{h}$. With this purpose we first exhibit a useful representation for $b$ and some bounds. From now on, we denote by $C, C^{\prime}$ positive constants that do not depend on $h$.

Proposition 9. For $\tau_{h} \in \mathbb{X}_{h}$ and $\mathbf{v} \in \mathbb{M}$, we have

$$
b\left(\tau_{h}, \mathbf{v}\right)=-\sum_{e \in \mathcal{C}_{h}}\left(\left[\tau_{h}\right]_{e}, \mathbf{v}\right)_{e} .
$$

Proof. For any $\tau_{h}=\left(\theta_{h}, q_{h}\right) \in \mathbb{X}_{h}$ and $\mathbf{v} \in \mathbb{M}$, we have

$$
\begin{aligned}
b\left(\tau_{h}, \mathbf{v}\right) & =-\left(\theta_{h}, \operatorname{curl} \mathbf{v}\right)+\left(q_{h}, \operatorname{div} \mathbf{v}\right) \\
& =-\sum_{K \in \mathcal{T}_{h}}\left\{\left(\theta_{h}, \operatorname{curl} \mathbf{v}\right)_{K}-\left(q_{h}, \operatorname{div} \mathbf{v}\right)_{K}\right\} \\
& =\sum_{K \in \mathcal{T}_{h}}\left(\theta_{h} \mathbf{t}_{e}^{K}-q_{h} \mathbf{n}_{e}^{K}, \mathbf{v}\right)_{\partial K} \\
& =-\sum_{e \in \mathcal{C}_{h}}\left(\left[\tau_{h}\right]_{e}, \mathbf{v}\right)_{e} .
\end{aligned}
$$


Corollary 10. There is a positive constant $C$, independent of $h$, such that:

- $\left|b\left(\tau_{h}, \mathbf{v}\right)\right| \leq C\left|\tau_{h}\right|_{h}\left\{\sum_{e \in \mathcal{C}_{h}} h_{e}^{-1}\|\mathbf{v}\|_{0, e}^{2}\right\}^{\frac{1}{2}}, \quad \forall \tau_{h} \in \mathbb{X}_{h}, \mathbf{v} \in \mathbb{M}$,

- $\left|b\left(\tau_{h}, \mathbf{v}\right)\right| \leq C\left|\tau_{h}\right|_{h}|\mathbf{v}|_{1, \Omega}, \quad \forall \tau_{h} \in \mathbb{V}_{h}, \mathbf{v} \in \mathbb{M} \cap \mathbb{H}^{1}(\Omega)$,

- $\left\|\operatorname{curl} \theta_{h}+\nabla q_{h}\right\|_{-1} \leq C\left|\tau_{h}\right|_{h}, \quad \forall \tau_{h}=\left(\theta_{h}, q_{h}\right) \in \mathbb{V}_{h}$.

Proof. The first estimate is an immediate consequence of Proposition 9 , Consider now $\tau_{h}=\left(\theta_{h}, q_{h}\right) \in \mathbb{V}_{h}$ and $\mathbf{v} \in \mathbb{M} \cap \mathbb{H}^{1}(\Omega)$. For any $\mathbf{v}_{h} \in \mathbb{M}_{h}$, we have $b\left(\tau_{h}, \mathbf{v}_{h}\right)=0$. Then, using Proposition 9, we deduce that

$$
\begin{aligned}
\left|b\left(\tau_{h}, \mathbf{v}-\mathbf{v}_{h}\right)\right| & =\left|\sum_{e \in \mathcal{C}_{h}}\left(\left[\tau_{h}\right]_{e}, \mathbf{v}-\mathbf{v}_{h}\right)_{e}\right| \\
& \leq \sum_{e \in \mathcal{C}_{h}}\left\|\left[\tau_{h}\right]_{e}\right\|_{0, e}\left\|\mathbf{v}-\mathbf{v}_{h}\right\|_{0, e} \\
& \leq\left|\tau_{h}\right|_{h}\left\{\sum_{e \in \mathcal{C}_{h}} \frac{1}{h_{e}}\left\|\mathbf{v}-\mathbf{v}_{h}\right\|_{0, e}^{2}\right\}^{\frac{1}{2}} .
\end{aligned}
$$

Now, for $\mathbf{v} \in \mathbb{M} \cap \mathbb{H}^{1}(\Omega)$ and $\mathbf{v}_{h} \in \mathbb{M}_{h}$ (see for example [4] among others), we have

$$
\left\{\sum_{e \in \mathcal{C}_{h}} \frac{1}{h_{e}}\left\|\mathbf{v}-\mathbf{v}_{h}\right\|_{0, e}^{2}\right\}^{\frac{1}{2}} \leq C|\mathbf{v}|_{1, \Omega}
$$

and the second relation of (3.14) is satisfied. In addition, by definition

$$
\left\|\operatorname{curl} \theta_{h}+\nabla q_{h}\right\|_{-1, \Omega}=\sup _{\mathbf{v} \in \mathbb{H}_{0}^{1}(\Omega)} \frac{\left|b\left(\tau_{h}, \mathbf{v}\right)\right|}{|\mathbf{v}|_{1, \Omega}} \leq C\left|\tau_{h}\right|_{h} .
$$

The following corollary ensures the coercivity of the bilinear form $a_{h}$.

Corollary 11. There is a positive constant $C$, independent of $h$, such that

$$
\left\|q_{h}\right\|_{0, \Omega} \leq C\left(\left|\tau_{h}\right|_{h}+\left\|\theta_{h}\right\|_{0, \Omega}\right), \quad \forall \tau_{h}=\left(\theta_{h}, q_{h}\right) \in \mathbb{V}_{h} .
$$

Proof. Consider $\tau_{h}=\left(\theta_{h}, q_{h}\right) \in \mathbb{V}_{h}$ and $\tilde{q}_{h}=q_{h}-\frac{1}{|\Omega|} \int_{\Omega} q_{h} d \Omega$. Then, $\tilde{q}_{h} \in L_{0}^{2}(\Omega)$. Moreover, using (2.8), we deduce that

$$
C\left\|\widetilde{q}_{h}\right\|_{0, \Omega} \leq\left\|\nabla \widetilde{q}_{h}\right\|_{-1, \Omega}=\left\|\nabla q_{h}\right\|_{-1, \Omega} \leq\left\|\operatorname{curl} \theta_{h}\right\|_{-1, \Omega} .
$$

Hence, it follows that

$$
C\left\|\widetilde{q}_{h}\right\|_{0, \Omega} \leq\left\|\theta_{h}\right\|_{0, \Omega} \leq C^{\prime}\left(\left|\tau_{h}\right|_{h}+\left\|\theta_{h}\right\|_{0, \Omega}\right) .
$$

In the case where $\left|\Gamma_{2}\right|=0$ we have $\widetilde{q}_{h}=q_{h}$, and therefore the proof is achieved. In the case where $\left|\Gamma_{2}\right|>0$, we consider a function $\mathbf{w} \in \mathbb{M} \cap \mathbb{H}^{1}(\Omega)$ such that $\int_{\Omega} \operatorname{div} \mathbf{w} d \Omega=1$. Then, we have

$$
\begin{aligned}
\frac{1}{|\Omega|}\left|\int_{\Omega} q_{h} d \Omega\right| & =\left|\int_{\Omega}\left(q_{h}-\widetilde{q}_{h}\right) \operatorname{div} \mathbf{w} d \Omega\right| \\
& =\left|b\left(\tau_{h}, \mathbf{w}\right)+\int_{\Omega} \theta_{h} \operatorname{curl} \mathbf{w} d \Omega-\int_{\Omega_{h}} \widetilde{q}_{h} \operatorname{div} \mathbf{w} d \Omega\right| \\
& \leq\left|b\left(\tau_{h}, \mathbf{w}\right)\right|+\left\{\left\|\theta_{h}\right\|_{0, \Omega}^{2}+\left\|\widetilde{q}_{h}\right\|_{0, \Omega}^{2}\right\}^{\frac{1}{2}}|\mathbf{w}|_{\mathbb{M}} .
\end{aligned}
$$


Moreover, using (3.14), we obtain

$$
\left|b\left(\tau_{h}, \mathbf{w}\right)\right| \leq C\left|\tau_{h}\right|_{h}|\mathbf{w}|_{1, \Omega} .
$$

It follows that

$$
\begin{aligned}
\frac{1}{|\Omega|}\left|\int_{\Omega} q_{h} d \Omega\right| & \leq C\left\{\left\|\theta_{h}\right\|_{0, \Omega}^{2}+\left\|\widetilde{q}_{h}\right\|_{0, \Omega}^{2}+\left|\tau_{h}\right|_{h}^{2}\right\}^{\frac{1}{2}}|\mathbf{w}|_{1, \Omega} \\
& \leq C\left\{\left\|\theta_{h}\right\|_{0, \Omega}^{2}+\left|\tau_{h}\right|_{h}^{2}\right\}^{\frac{1}{2}}|\mathbf{w}|_{1, \Omega} .
\end{aligned}
$$

The proof is then achieved using

$$
\left\|q_{h}\right\|_{0, \Omega} \leq\left\|\widetilde{q}_{h}\right\|_{0, \Omega}+\frac{1}{\sqrt{|\Omega|}}\left|\int_{\Omega} q_{h} d \Omega\right| .
$$

Remark 3. Corollary 11 states the coercivity of the bilinear form $a_{h}$ on the discrete kernel $\mathbb{V}_{h}$. Indeed, for $\tau_{h}=\left(\theta_{h}, q_{h}\right) \in \mathbb{V}_{h}$, we have

$$
\begin{aligned}
a_{h}\left(\tau_{h}, \tau_{h}\right) & =\left\|\theta_{h}\right\|_{0, \Omega}^{2}+\beta_{h}\left|\tau_{h}\right|_{h}^{2} \\
& \geq \frac{1}{2}\left\|\theta_{h}\right\|_{0, \Omega}^{2}+\min \left\{\frac{1}{2}, \beta_{h}\right\}\left\{\left\|\theta_{h}\right\|_{0, \Omega}^{2}+\left|\tau_{h}\right|_{h}^{2}\right\} \\
& \geq \frac{1}{2}\left\|\theta_{h}\right\|_{0, \Omega}^{2}+\min \left\{\frac{1}{2 C}, \frac{\beta_{h}}{C}\right\}\left\|q_{h}\right\|_{0, \Omega}^{2} .
\end{aligned}
$$

We note that the constant of coercivity depends on $\beta_{h}$, since we have

$$
a_{h}\left(\tau_{h}, \tau_{h}\right) \geq \min \left\{\frac{1}{2}, \frac{1}{2 C}, \frac{\beta_{h}}{C}\right\}\left\|\tau_{h}\right\|_{\mathbb{X}}^{2} \quad \forall \tau_{h} \in \mathbb{V}_{h} .
$$

Next, we prove the continuity of the form $A_{h}$ on $\mathbb{X}_{h} \times \mathbb{X}_{h}$ in order to deduce the continuity of the form $a_{h}$. This property will be used later in order to prove the consistency of the new terms added to the formulation.

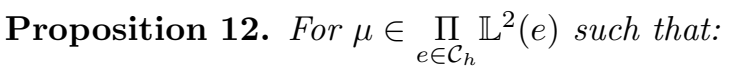

$$
\begin{aligned}
& \text { - } \mu=\mathbf{0} \text { on } e, \quad \text { if } e \subset \Gamma_{1} \text {, } \\
& \text { - } \mu . \mathbf{t}=0 \text { on } e, \quad \text { if } e \subset \Gamma_{2} \text {, } \\
& \text { - } \mu \cdot \mathbf{n}=0 \text { on } e, \quad \text { if } e \subset \Gamma_{3} \text {, }
\end{aligned}
$$

there is a function $\boldsymbol{\Phi}_{h} \in \mathbb{M} \cap \mathbb{H}^{1}(\Omega)$ such that

$$
\begin{aligned}
& \text { - } b\left(\tau_{h}, \boldsymbol{\Phi}_{h}\right)=-\sum_{e \in \mathcal{C}_{h}}\left(\left[\tau_{h}\right]_{e}, \mu\right)_{e}, \\
& \text { - }\left|\boldsymbol{\Phi}_{h}\right|_{1, \Omega} \leq C\left\{\sum_{e \in \mathcal{C}_{h}} \frac{1}{h_{e}}\|\mu\|_{0, e}^{2}\right\}^{\frac{1}{2}},
\end{aligned}
$$

for all $\tau_{h}=\left(\theta_{h}, q_{h}\right) \in \mathbb{X}_{h}$.

Proof. We consider the function $\boldsymbol{\Phi}_{h}$ satisfying

- $\left.\boldsymbol{\Phi}_{h}\right|_{K} \in \mathbb{P}_{2}(K), \quad \forall K \in \mathcal{T}_{h}$,

- $\boldsymbol{\Phi}_{h}(S)=\mathbf{0}, \quad \forall S$ vertex of $\mathcal{T}_{h}$,

- $\int_{e} \mathbf{\Phi}_{h} d \sigma=\int_{e} \mu d \sigma, \quad \forall e$ edge of $\mathcal{T}_{h}$. 
Similarly to the proof of Proposition 9, we have

$$
\begin{aligned}
b\left(\tau_{h}, \boldsymbol{\Phi}_{h}\right) & =-\left(\theta_{h}, \operatorname{curl} \mathbf{\Phi}_{h}\right)+\left(q_{h}, \operatorname{div} \boldsymbol{\Phi}_{h}\right) \\
& =-\sum_{e \in \mathcal{C}_{h}}\left(\left[\tau_{h}\right]_{e}, \mathbf{\Phi}_{h}\right)_{e} \\
& =-\sum_{e \in \mathcal{C}_{h}}\left(\left[\tau_{h}\right]_{e}, \mu\right)_{e},
\end{aligned}
$$

for $\tau_{h}=\left(\theta_{h}, q_{h}\right) \in \mathbb{X}_{h}$. Then, it follows that

$$
\left|b\left(\tau_{h}, \boldsymbol{\Phi}_{h}\right)\right| \leq\left|\tau_{h}\right|_{h}\left\{\sum_{e \in \mathcal{C}_{h}} \frac{1}{h_{e}}\|\mu\|_{0, e}^{2}\right\}^{\frac{1}{2}} .
$$

Finally, from the classical inverse inequalities, we have the existence of two positive constants $\widehat{c}_{0}$ and $\widehat{c}_{1}$, independent of $h$, such that

$$
\widehat{c}_{0}\left|\mathbf{\Phi}_{h}\right|_{1, K} \leq\left\{\sum_{e \subset \partial K} \frac{1}{h_{e}}\|\mu\|_{0, e}^{2}\right\}^{\frac{1}{2}} \leq \widehat{c}_{1}\left|\Phi_{h}\right|_{1, K}, \quad \forall K \in \mathcal{T}_{h} .
$$

Therefore, we conclude the proof by setting $\tau_{h}=\mathbf{\Phi}_{h}$.

Corollary 13. For $\tau_{h} \in \mathbb{X}_{h}$, there is a function $\Phi_{h} \in \mathbb{M} \cap \mathbb{H}^{1}(\Omega)$ satisfying

$$
b\left(\delta_{h}, \boldsymbol{\Phi}_{h}\right)=A_{h}\left(\delta_{h}, \tau_{h}\right) \quad \forall \delta_{h} \in \mathbb{X}_{h} \quad \text { and } \quad\left|\boldsymbol{\Phi}_{h}\right|_{1, \Omega} \leq C\left|\tau_{h}\right|_{h} .
$$

In addition, if we write $\tau_{h}=\left(\theta_{h}, q_{h}\right)$, we have

$$
\left|\tau_{h}\right|_{h} \leq C\left\{\left\|\theta_{h}\right\|_{0, \Omega}^{2}+\left\|q_{h}\right\|_{0, \Omega}^{2}\right\}^{\frac{1}{2}}=C\left\|\tau_{h}\right\|_{\mathbb{X}} \cdot
$$

Proof. We apply Proposition 12 for $\mu \in \prod_{e \in \mathcal{C}_{h}} \mathbb{L}^{2}(e)$ given by

$$
\left.\mu\right|_{e}=-h_{e}\left[\tau_{h}\right]_{e} .
$$

It follows that for $\delta_{h} \in \mathbb{X}_{h}$, we have

$$
\begin{aligned}
A_{h}\left(\delta_{h}, \tau_{h}\right) & =\sum_{e \in \mathcal{C}_{h}} h_{e}\left(\left[\delta_{h}\right]_{e},\left[\tau_{h}\right]_{e}\right)_{e} \\
& =-\sum_{e \in \mathcal{C}_{h}}\left(\left[\delta_{h}\right]_{e}, \mu\right)_{e} \\
& =b\left(\delta_{h}, \boldsymbol{\Phi}_{h}\right) .
\end{aligned}
$$

Then,

$$
\begin{aligned}
\left|\tau_{h}\right|_{h}^{2} & =b\left(\tau_{h}, \boldsymbol{\Phi}_{h}\right)=-\left(\theta_{h}, \operatorname{curl} \boldsymbol{\Phi}_{h}\right)+\left(q_{h}, \operatorname{div} \boldsymbol{\Phi}_{h}\right) \\
& \leq\left\{\left\|\theta_{h}\right\|_{0, \Omega}^{2}+\left\|q_{h}\right\|_{0, \Omega}^{2}\right\}^{\frac{1}{2}}\left|\mathbf{\Phi}_{h}\right|_{\mathbb{M}} \leq C\left\|\tau_{h}\right\|_{\mathbb{X}}\left|\mathbf{\Phi}_{h}\right|_{1, \Omega} \leq C\left\|\tau_{h}\right\|_{\mathbb{X}}\left|\tau_{h}\right|_{h} .
\end{aligned}
$$

The a priori estimate (3.18) is then established. Consequently, the bilinear form $A_{h}$ is continuous on $\mathbb{X}_{h} \times \mathbb{X}_{h}$.

Next, we define the $L^{2}$-projections of the functions $p_{0}$ and $\omega_{0}$ as follows:

$$
\begin{aligned}
& \left\{\begin{array}{l}
p_{0_{h}} \in L^{2}\left(\Gamma_{2}\right) \quad \text { and } \forall e \in \mathcal{F}_{h}^{2}, \\
\left.p_{0_{h}}\right|_{e} \in P_{0}(e), \quad\left(p_{0}-p_{0_{h}}, y_{h}\right)_{K}=0, \quad \forall y_{h} \in P_{0}(e) m,
\end{array}\right. \\
& \left\{\begin{array}{l}
\omega_{0_{h}} \in L^{2}\left(\Gamma_{3}\right) \quad \text { and } \forall e \in \mathcal{F}_{h}^{3}, \\
\left.\omega_{0_{h}}\right|_{e} \in P_{0}(e), \quad\left(\omega_{0}-\omega_{0_{h}}, y_{h}\right)_{K}=0, \quad \forall y_{h} \in P_{0}(e) .
\end{array}\right.
\end{aligned}
$$


We are now ready to establish the consistency of the additional terms in the formulation. We have

Proposition 14. Let $\sigma=(\omega, p)$ be the solution of the continuous problem (2.7). There is a constant $C>0$, independent of $h$, such that for a given $\tau_{h} \in \mathbb{X}_{h}$ and $\delta_{h} \in \mathbb{X}_{h}$ we have

$$
\left|A_{h}\left(\delta_{h}, \tau_{h}\right)-G_{h}\left(\tau_{h}\right)\right| \leq C\left|\tau_{h}\right|_{h}\left\{\begin{array}{l}
\left\|\sigma-\delta_{h}\right\|_{\mathbb{X}}+h\|\mathbf{f}\|_{0, \Omega} \\
+\sqrt{h}\left\|p_{0}-p_{0_{h}}\right\|_{0, \Gamma_{2}}+\sqrt{h}\left\|\omega_{0}-\omega_{0_{h}}\right\|_{0, \Gamma_{3}}
\end{array}\right\} .
$$

Proof. Similarly to Corollary [13, for given $\tau_{h}=\left(\theta_{h}, q_{h}\right) \in \mathbb{X}_{h}$ and $\delta_{h}=\left(\rho_{h}, r_{h}\right) \in$ $\mathbb{X}_{h}$, we consider the function $\boldsymbol{\Phi}_{h}$ associated to $\tau_{h}$. Then, we have

$$
A_{h}\left(\delta_{h}, \tau_{h}\right)=b\left(\delta_{h}, \boldsymbol{\Phi}_{h}\right) \quad \text { and } \quad\left|\boldsymbol{\Phi}_{h}\right|_{1, \Omega} \leq C\left|\tau_{h}\right|_{h} .
$$

Furthermore, we have

$$
A_{h}\left(\delta_{h}, \tau_{h}\right)-G_{h}\left(\tau_{h}\right)=b\left(\delta_{h}-\sigma, \mathbf{\Phi}_{h}\right)-F\left(\mathbf{\Phi}_{h}\right)-G_{h}\left(\tau_{h}\right)
$$

with $F\left(\mathbf{v}_{h}\right)=\left(\mathbf{f}, \mathbf{v}_{h}\right)+\left\langle\omega_{0}, \mathbf{v}_{h} \cdot \mathbf{t}\right\rangle_{\Gamma_{3}}-\left\langle p_{0}, \mathbf{v}_{h} \cdot \mathbf{n}\right\rangle_{\Gamma_{2}}$ and

$$
\begin{aligned}
G_{h}\left(\tau_{h}\right)=-\sum_{e \in \mathcal{F}_{h}^{2}} h_{e}\left(p_{0},\left[q_{h}\right]_{e}\right)_{e}+\sum_{e \in \mathcal{F}_{h}^{3}} h_{e}\left(\omega_{0},\left[\theta_{h}\right]_{e}\right)_{e} \\
=-\sum_{e \in \mathcal{F}_{h}^{2}} h_{e}\left(p_{0_{h}},\left[q_{h}\right]_{e}\right)_{e}+\sum_{e \in \mathcal{F}_{h}^{3}} h_{e}\left(\omega_{0_{h}},\left[\theta_{h}\right]_{e}\right)_{e} \\
=-\sum_{e \in \mathcal{F}_{h}^{2}}\left(p_{0_{h}}, \boldsymbol{\Phi}_{h} \cdot \mathbf{n}_{e}\right)_{e}+\sum_{e \in \mathcal{F}_{h}^{3}}\left(\omega_{0_{h}}, \boldsymbol{\Phi}_{h} \cdot \mathbf{t}_{e}\right)_{e} \\
=-\left\langle p_{0_{h}}, \boldsymbol{\Phi}_{h} \cdot \mathbf{n}\right\rangle_{\Gamma_{2}}+\left\langle\omega_{0_{h}}, \boldsymbol{\Phi}_{h} \cdot \mathbf{t}\right\rangle_{\Gamma_{3}} .
\end{aligned}
$$

It follows that

$$
\begin{aligned}
\left|F\left(\mathbf{\Phi}_{h}\right)+G_{h}\left(\tau_{h}\right)\right|= & \left|\left(\mathbf{f}, \boldsymbol{\Phi}_{h}\right)-\left\langle p_{0}-p_{0_{h}}, \boldsymbol{\Phi}_{h} \cdot \mathbf{n}\right\rangle_{\Gamma_{2}}+\left\langle\omega_{0}-\omega_{0_{h}}, \boldsymbol{\Phi}_{h} \cdot \mathbf{t}\right\rangle_{\Gamma_{3}}\right| \\
\leq & \|\mathbf{f}\|_{0, \Omega}\left\|\mathbf{\Phi}_{h}\right\|_{0, \Omega}+\left\|p_{0}-p_{0_{h}}\right\|_{0, \Gamma_{2}}\left\|\boldsymbol{\Phi}_{h}\right\|_{0, \Gamma_{2}} \\
& +\left\|\omega_{0}-\omega_{0_{h}}\right\|_{0, \Gamma_{3}}\left\|\boldsymbol{\Phi}_{h}\right\|_{0, \Gamma_{3}} .
\end{aligned}
$$

Using classical inverse inequalities and the fact that the function $\Phi_{h}$ vanishes at each vertex of $\mathcal{T}_{h}$, we deduce that

$$
\left\|\boldsymbol{\Phi}_{h}\right\|_{0, \Omega} \leq C h\left|\mathbf{\Phi}_{h}\right|_{1, \Omega} \leq C^{\prime} h\left|\tau_{h}\right|_{h} \quad \text { and }\left\|\boldsymbol{\Phi}_{h}\right\|_{0, \Gamma} \leq C \sqrt{h}\left|\boldsymbol{\Phi}_{h}\right|_{1, \Omega} \leq C^{\prime} \sqrt{h}\left|\tau_{h}\right|_{h} .
$$

Moreover, we have

$$
\begin{aligned}
\left|b\left(\delta_{h}-\sigma, \mathbf{\Phi}_{h}\right)\right| & \leq C\left\|\mathbf{\Phi}_{h}\right\|_{\mathbb{M}}\left\|\sigma-\delta_{h}\right\|_{\mathbb{X}} \\
& \leq C\left|\tau_{h}\right|_{h}\left\|\sigma-\delta_{h}\right\|_{\mathbb{X}},
\end{aligned}
$$

which achieves the proof of this proposition.

Remark 4. We note that if $\delta_{h}$ is a good approximation of $\sigma$, the added terms introduce an error which is of the same order as the one expected when using finite element methods. 


\section{ERror estimates}

Our aim here is to estimate the error $\left\|\sigma-\sigma_{h}\right\|_{\mathbb{X}}+\left\|\mathbf{u}-\mathbf{u}_{h}\right\|_{\mathbb{M} \text { when }}(\sigma=(\omega, p), \mathbf{u})$ is the solution of the continuous problem (2.7) and $\left(\sigma_{h}=\left(\left(\omega_{h}, p_{h}\right), \mathbf{u}_{h}\right)\right.$ is the solution of the discrete one (3.12). We point out that, from the standard analysis, one can establish these error estimates directly. Our objective here is to derive more precise estimates. To do this, we introduce

$$
\begin{aligned}
& \bar{\sigma}_{h}=\left(\bar{\omega}_{h}, \bar{p}_{h}\right) \in \mathbb{X}_{h} \text { such that, } \quad \forall K \in \mathcal{T}_{h}, \\
& \begin{cases}\left.\bar{p}_{h}\right|_{K} \in P_{0}(K), \quad\left(p-\bar{p}_{h}, q_{h}\right)_{K}=0, \quad \forall q_{h} \in P_{0}(K), \\
\left.\bar{\omega}_{h}\right|_{K} \in P_{0}(K), \quad\left(\omega-\bar{\omega}_{h}, \theta_{h}\right)_{K}=0, \quad \forall \theta_{h} \in P_{0}(K),\end{cases}
\end{aligned}
$$

i.e., $\bar{\sigma}_{h}$ is the $\mathbb{L}^{2}(\Omega)$ projection of $\sigma$ on $\mathbb{X}_{h}$. Moreover, we have

$$
\begin{cases}a\left(\sigma-\bar{\sigma}_{h}, \tau_{h}\right)=0, & \forall \tau_{h} \in \mathbb{X}_{h} \\ b\left(\sigma-\bar{\sigma}_{h}, \mathbf{v}_{h}\right)=0, & \forall \mathbf{v}_{h} \in \mathbb{M}_{h} .\end{cases}
$$

From problem (2.7), we deduce that

$$
\begin{cases}a\left(\sigma, \tau_{h}\right)+b\left(\tau_{h}, \mathbf{u}\right)=0, & \forall \tau_{h} \in \mathbb{X}_{h} \\ b\left(\sigma, \mathbf{v}_{h}\right)=-F\left(\mathbf{v}_{h}\right), & \forall \mathbf{v}_{h} \in \mathbb{M}_{h} .\end{cases}
$$

Then

$$
\begin{cases}a\left(\bar{\sigma}_{h}, \tau_{h}\right)+b\left(\tau_{h}, \mathbf{u}\right)=0, & \forall \tau_{h} \in \mathbb{X}_{h} \\ b\left(\bar{\sigma}_{h}, \mathbf{v}_{h}\right)=-F\left(\mathbf{v}_{h}\right), & \forall \mathbf{v}_{h} \in \mathbb{M}_{h} .\end{cases}
$$

Using problem (3.12), we obtain

$$
\begin{cases}a\left(\bar{\sigma}_{h}-\sigma_{h}, \tau_{h}\right)-\beta_{h} A_{h}\left(\sigma_{h}, \tau_{h}\right)+b\left(\tau_{h}, \mathbf{u}-\mathbf{u}_{h}\right)=-\beta_{h} G_{h}\left(\tau_{h}\right), & \forall \tau_{h} \in \mathbb{X}_{h}, \\ b\left(\bar{\sigma}_{h}-\sigma_{h}, \mathbf{v}_{h}\right)=0, & \forall \mathbf{v}_{h} \in \mathbb{M}_{h} .\end{cases}
$$

We set

$$
\delta_{h}=\bar{\sigma}_{h}-\sigma_{h}=\left(\bar{\omega}_{h}-\omega_{h}, \bar{p}_{h}-p_{h}\right) .
$$

Hence, we have

$$
\begin{cases}a_{h}\left(\delta_{h}, \tau_{h}\right)+b\left(\tau_{h}, \mathbf{u}-\mathbf{u}_{h}\right)=\beta_{h}\left\{A_{h}\left(\bar{\sigma}_{h}, \tau_{h}\right)-G_{h}\left(\tau_{h}\right)\right\}, & \forall \tau_{h} \in \mathbb{X}_{h}, \\ b\left(\delta_{h}, \mathbf{v}_{h}\right)=0, & \forall \mathbf{v}_{h} \in \mathbb{M}_{h},\end{cases}
$$

with $a_{h}\left(\delta_{h}, \tau_{h}\right)=a\left(\delta_{h}, \tau_{h}\right)+\beta_{h} A_{h}\left(\delta_{h}, \tau_{h}\right)$. We remark that $\delta_{h} \in \mathbb{V}_{h}$. We have

Proposition 15. For $\mathbf{v}_{h} \in \mathbb{M}_{h}$

$$
\left\|\bar{\omega}_{h}-\omega_{h}\right\|_{0, \Omega}^{2}+\beta_{h}\left|\delta_{h}\right|_{h}^{2}=b\left(\delta_{h}, \mathbf{v}_{h}-\mathbf{u}\right)+\beta_{h}\left\{A_{h}\left(\bar{\sigma}_{h}, \delta_{h}\right)-G_{h}\left(\delta_{h}\right)\right\} .
$$

Proof. We apply (4.4) for $\tau_{h}=\delta_{h}$ given by (4.3). Then, for $\mathbf{v}_{h} \in \mathbb{M}_{h}$, we have

$$
\begin{aligned}
a_{h}\left(\delta_{h}, \delta_{h}\right) & =b\left(\delta_{h}, \mathbf{u}_{h}-\mathbf{u}\right)+\beta_{h}\left(A_{h}\left(\bar{\sigma}_{h}, \delta_{h}\right)-G_{h}\left(\delta_{h}\right)\right) \\
& =b\left(\delta_{h}, \mathbf{v}_{h}-\mathbf{u}\right)+\beta_{h}\left(A_{h}\left(\bar{\sigma}_{h}, \delta_{h}\right)-G_{h}\left(\delta_{h}\right)\right) .
\end{aligned}
$$

The proof is then achieved by using

$$
a_{h}\left(\delta_{h}, \delta_{h}\right)=\left\|\bar{\omega}_{h}-\omega_{h}\right\|_{0, \Omega}^{2}+\beta_{h}\left|\delta_{h}\right|_{h}^{2} .
$$

We denote by $E_{c}$ the error of consistency, defined as follows:

$$
E_{c}=\left\{\begin{array}{l}
\left\|\sigma-\bar{\sigma}_{h}\right\|_{\mathbb{X}}+h\|\mathbf{f}\|_{0, \Omega} \\
+\sqrt{h}\left\|p_{0}-p_{0_{h}}\right\|_{0, \Gamma_{2}}+\sqrt{h}\left\|\omega_{0}-\omega_{0_{h}}\right\|_{0, \Gamma_{3}}
\end{array}\right\} .
$$


Then, from Lemma 14, we have the existence of a constant $C>0$, independent of $h$, such that

$$
\left|A_{h}\left(\bar{\sigma}_{h}, \delta_{h}\right)-G_{h}\left(\delta_{h}\right)\right| \leq C E_{c}\left|\delta_{h}\right|_{h} .
$$

We also have from definition (2.6) that

$$
\left|b\left(\delta_{h}, \mathbf{v}_{h}-\mathbf{u}\right)\right| \leq\left|\mathbf{u}-\mathbf{v}_{h}\right|_{\mathbb{M}}\left\|\delta_{h}\right\|_{\mathbb{X}} .
$$

Hence we deduce from Proposition 15 and these two previous estimates the following first error estimate:

Lemma 16. We set $\bar{\beta}_{h}=\min \left(1, \beta_{h}\right)$ and denote the error on $\sigma$ by

$$
E_{h}(\sigma)=\left\|\bar{\omega}_{h}-\omega_{h}\right\|_{0, \Omega}+\sqrt{\bar{\beta}_{h}}\left\|\bar{p}_{h}-p_{h}\right\|_{0, \Omega}+\sqrt{\beta_{h}}\left|\delta_{h}\right|_{h} .
$$

Then, there is a constant $C>0$, independent of $h$, such that for $\mathbf{v}_{h} \in \mathbb{M}_{h}$, we have

$$
E_{h}(\sigma) \leq \frac{C}{\sqrt{\bar{\beta}_{h}}}\left|\mathbf{u}-\mathbf{v}_{h}\right|_{\mathbb{M}}+C \sqrt{\beta_{h}} E_{c} .
$$

Proof. Relation (3.15) gives the following inequality:

$$
\left\|\bar{p}_{h}-p_{h}\right\|_{0, \Omega} \leq C\left(\left|\delta_{h}\right|_{h}+\left\|\bar{\omega}_{h}-\omega_{h}\right\|_{0, \Omega}\right) .
$$

Then, we have

$$
\begin{aligned}
\left\|\bar{\omega}_{h}-\omega_{h}\right\|_{0, \Omega}+\sqrt{\beta_{h}}\left|\delta_{h}\right|_{h} & \geq \sqrt{\bar{\beta}_{h}}\left\{\left\|\bar{\omega}_{h}-\omega_{h}\right\|_{0, \Omega}+\left|\delta_{h}\right|_{h}\right\} \\
& \geq C \sqrt{\bar{\beta}_{h}}\left\|\delta_{h}\right\|_{\mathbb{X}} .
\end{aligned}
$$

Using (4.5)), we deduce that

$$
\begin{aligned}
\left\|\bar{\omega}_{h}-\omega_{h}\right\|_{0, \Omega}^{2} & +\beta_{h}\left|\delta_{h}\right|_{h}^{2}+\bar{\beta}_{h}\left\|\delta_{h}\right\|_{\mathbb{X}}^{2} \\
& \leq C\left|b\left(\delta_{h}, \mathbf{v}_{h}-\mathbf{u}\right)\right|+C \beta_{h}\left|A_{h}\left(\bar{\sigma}_{h}, \delta_{h}\right)-G_{h}\left(\delta_{h}\right)\right| .
\end{aligned}
$$

Proposition 17. There is a constant $C>0$, independent of $h$, such that for $\mathbf{v}_{h} \in \mathbb{M}_{h}$ we have the following error estimate:

$$
\left|\mathbf{u}-\mathbf{u}_{h}\right|_{\mathbb{M}} \leq C\left\{E_{c}+\max \left(1, \sqrt{\beta_{h}}\right) E_{h}(\sigma)+\left|\mathbf{u}-\mathbf{v}_{h}\right|_{\mathbb{M}}\right\} .
$$

Proof. For $\mathbf{v}_{h} \in \mathbb{M}_{h}$ we define

$$
\bar{\tau}_{h}=\left(-\operatorname{curl}\left(\mathbf{u}_{h}-\mathbf{v}_{h}\right), \operatorname{div}\left(\mathbf{u}_{\mathbf{h}}-\mathbf{v}_{\mathbf{h}}\right)\right) \in \mathbb{X}_{h} .
$$

Hence, we have

$$
b\left(\bar{\tau}_{h}, \mathbf{u}_{h}-\mathbf{v}_{h}\right)=\left|\mathbf{u}_{h}-\mathbf{v}_{h}\right|_{\mathbb{M}}^{2}=\left\|\bar{\tau}_{h}\right\|_{\mathbb{X}}^{2}
$$

and

$$
b\left(\bar{\tau}_{h}, \mathbf{u}_{h}-\mathbf{v}_{h}\right)=b\left(\bar{\tau}_{h}, \mathbf{u}_{h}-\mathbf{u}\right)+b\left(\bar{\tau}_{h}, \mathbf{u}-\mathbf{v}_{h}\right) .
$$

Using (4.4), we deduce that

$$
b\left(\bar{\tau}_{h}, \mathbf{u}-\mathbf{u}_{h}\right)=\beta_{h}\left\{A_{h}\left(\bar{\sigma}_{h}, \bar{\tau}_{h}\right)-G_{h}\left(\bar{\tau}_{h}\right)\right\}-a_{h}\left(\delta_{h}, \bar{\tau}_{h}\right),
$$

which implies that

$$
\begin{aligned}
\left|b\left(\bar{\tau}_{h}, \mathbf{u}-\mathbf{u}_{h}\right)\right| \leq & \beta_{h}\left|A_{h}\left(\bar{\sigma}_{h}, \bar{\tau}_{h}\right)-G_{h}\left(\bar{\tau}_{h}\right)\right| \\
& +\left\|\bar{\omega}_{h}-\omega_{h}\right\|_{0, \Omega}\left\|\operatorname{curl}\left(\mathbf{u}_{h}-\mathbf{v}_{h}\right)\right\|_{0, \Omega}+\beta_{h}\left|\delta_{h}\right|_{h}\left|\bar{\tau}_{h}\right|_{h} .
\end{aligned}
$$

Moreover, since (3.18) states that

$$
\left|\bar{\tau}_{h}\right|_{h} \leq C\left\|\bar{\tau}_{h}\right\|_{\mathbb{X}}
$$


and since

it follows that

$$
\left|b\left(\bar{\tau}_{h}, \mathbf{u}-\mathbf{v}_{h}\right)\right| \leq\left|\mathbf{u}_{h}-\mathbf{v}_{h}\right|_{\mathbb{M}}\left|\mathbf{u}-\mathbf{v}_{h}\right|_{\mathbb{M}}
$$

$$
\begin{aligned}
\mid \mathbf{u}- & \left.\mathbf{u}_{h}\right|_{\mathbb{M}} \\
& \leq C\left\{\frac{\beta_{h}\left|A_{h}\left(\bar{\sigma}_{h}, \bar{\tau}_{h}\right)-G_{h}\left(\bar{\tau}_{h}\right)\right|}{\left|\bar{\tau}_{h}\right|_{h}}+\left\|\bar{\omega}_{h}-\omega_{h}\right\|_{0, \Omega}+\beta_{h}\left|\delta_{h}\right|_{h}+\left|\mathbf{u}-\mathbf{v}_{h}\right|_{\mathbb{M}}\right\} .
\end{aligned}
$$

Therefore, (4.9) is an immediate consequence.

We conclude this section with the following global error estimates:

Theorem 18. Let $E_{h}$ be the global error defined by

$$
E_{h}=\left\|\omega-\omega_{h}\right\|_{0, \Omega}+\sqrt{\bar{\beta}_{h}}\left\|p-p_{h}\right\|_{0, \Omega}+\left|\mathbf{u}-\mathbf{u}_{h}\right|_{\mathbb{M}} .
$$

Then there is a constant $C>0$, independent of $h$, such that for $\mathbf{v}_{h} \in \mathbb{M}_{h}$ we have

$$
E_{h} \leq C\left\{\begin{array}{l}
\max \left(1, \beta_{h}\right) E_{c}+\max \left(\frac{1}{\sqrt{\beta_{h}}}, \sqrt{\beta_{h}}\right)\left|\mathbf{u}-\mathbf{v}_{h}\right|_{\mathbb{M}} \\
+\left\|\omega-\bar{\omega}_{h}\right\|_{0, \Omega}+\sqrt{\bar{\beta}_{h}}\left\|p-\bar{p}_{h}\right\|_{0, \Omega}
\end{array}\right\} .
$$

The following result is an immediate consequence:

Theorem 19. We assume that $\beta_{h}=\beta$ is independent of $h$. Then, there is a constant $C>0$, dependent on $\beta$ and independent of $h$, such that for $\mathbf{v}_{h} \in \mathbb{M}_{h}$ we have

$$
\left\|\sigma-\sigma_{h}\right\|_{\mathbb{X}}+\left|\mathbf{u}-\mathbf{u}_{h}\right|_{\mathbb{M}} \leq C\left\{E_{c}+\left\|\sigma-\bar{\sigma}_{h}\right\|_{\mathbb{X}}+\left|\mathbf{u}-\mathbf{v}_{h}\right|_{\mathbb{M}}\right\} .
$$

Corollary 20. Under the assumption of Theorem 19, we have

- $\lim _{h \rightarrow 0} E_{h}=0$, i.e., the method is unconditionally convergent, and

- if $\mathbf{u} \in \mathbb{H}^{2}(\Omega), \omega \in H^{1}(\Omega)$ and $p \in H^{1}(\Omega)$, then $E_{h}=O(h)$, i.e., the method is optimal in terms of finite elements.

\section{NumericAl RESUlts}

We present in this section numerical results to illustrate the computational efficiency of the proposed formulation. We recall that we solve the following problem:

$$
\begin{cases}\text { Find }\left(\sigma_{h}, \mathbf{u}_{h}\right) \in \mathbb{X}_{h} \times \mathbb{M}_{h} \text { with } \sigma_{h}=\left(\omega_{h}, p_{h}\right) \quad \text { such that } \\ a_{h}\left(\sigma_{h}, \tau_{h}\right)+b\left(\tau_{h}, \mathbf{u}_{h}\right)=\beta G_{h}\left(\tau_{h}\right), & \forall \tau_{h}=\left(\theta_{h}, q_{h}\right) \in \mathbb{X}_{h}, \\ b\left(\sigma_{h}, \mathbf{v}_{h}\right)=-F\left(\mathbf{v}_{h}\right), & \forall \mathbf{v}_{h} \in \mathbb{M}_{h}\end{cases}
$$

where

$$
\begin{aligned}
a_{h}\left(\sigma_{h}, \tau_{h}\right) & =a\left(\sigma_{h}, \tau_{h}\right)+\beta A_{h}\left(\sigma_{h}, \tau_{h}\right)=\left(\omega_{h}, \theta_{h}\right)+\beta \sum_{e \in \mathcal{C}_{h}} h_{e}\left(\left[\sigma_{h}\right]_{e},\left[\tau_{h}\right]_{e}\right)_{e} \\
& =\left(\omega_{h}, \theta_{h}\right)+\beta \sum_{e \in \mathcal{C}_{h}} h_{e}\left(\left(\left[\omega_{h}\right]_{e},\left[\theta_{h}\right]_{e}\right)_{e}+\left(\left[p_{h}\right]_{e},\left[q_{h}\right]_{e}\right)_{e}\right)
\end{aligned}
$$

and

$$
G_{h}\left(\tau_{h}\right)=\sum_{e \in \mathcal{F}_{h}^{2}} h_{e}\left(p_{0},\left[q_{h}\right]_{e}\right)_{e}+\sum_{e \in \mathcal{F}_{h}^{3}} h_{e}\left(\omega_{0},\left[\theta_{h}\right]_{e}\right)_{e} .
$$

Test 1: An analytical test. First, we consider the Bercovier-Engelman test, since we are able to compute the solution analytically. Recall that when the exact solution satisfies $\mathbf{u} \in \mathbb{H}^{2}(\Omega), \omega \in H^{1}(\Omega)$ and $p \in H^{1}(\Omega)$, then $E_{h}=O(h)$. For this test, we have $\Omega=] 0,1\left[^{2}\right.$ and the following boundary conditions: $p$ and $u_{2}$ are 
TABLE 1.

\begin{tabular}{|c|c|c|c|c|c|}
\hline mesh & $5 \times 5$ & $10 \times 10$ & $20 \times 20$ & $40 \times 40$ & $80 \times 80$ \\
\hline error on $\omega$ in $L^{2}$-norm & 2.24 & 0.97 & 0.44 & 0.21 & 0.10 \\
\hline error on $p$ in $L^{2}$-norm & 3.26 & 1.81 & 0.93 & 0.47 & 0.23 \\
\hline error on $u_{1}$ in norm $|\cdot|_{1, \Omega}$ & 2.12 & 1.12 & 0.57 & 0.28 & 0.14 \\
\hline error on $u_{2}$ in norm $|\cdot|_{1, \Omega}$ & 2.04 & 1.03 & 0.51 & 0.25 & 0.13 \\
\hline
\end{tabular}

TABLE 2 .

\begin{tabular}{|c|c|c|c|c|}
\hline & $\beta=1$ & $\beta=0.1$ & $\beta=0.05$ & $\beta=0.01$ \\
\hline error on $\omega$ in $L^{2}$-norm & 0.57 & 0.2 & 0.2 & 0.32 \\
\hline error on $p$ in $L^{2}$-norm & 1.78 & 0.55 & 1.07 & 4.97 \\
\hline error on $u_{1}$ in norm $|\cdot|_{1, \Omega}$ & 1.06 & 0.24 & 0.23 & 0.27 \\
\hline error on $u_{2}$ in norm $|\cdot|_{1, \Omega}$ & 1.06 & 0.24 & 0.23 & 0.29 \\
\hline
\end{tabular}

prescribed on the right-hand side boundary, $\omega$ and $u_{1}$ are prescribed on the lefthand side boundary, and $\mathbf{u}=\mathbf{0}$ on the upper and lower boundary. The right-hand sides $f_{1}$ and $f_{2}$ of the equations are chosen so that the exact solution is

$$
\begin{aligned}
\omega(x, y) & =256\left(y^{2}(y-1)^{2}\left(6 x^{2}-6 x+1\right)+x^{2}(x-1)^{2}\left(6 y^{2}-6 y+1\right)\right), \\
u_{1}(x, y) & =-256 y(y-1)(2 y-1) x^{2}(x-1)^{2}, \\
u_{2}(x, y) & =-u_{1}(y, x), \\
p(x, y) & =(x-0.5)(y-0.5) .
\end{aligned}
$$

We report in Table 1 the results obtained on the absolute error in the $L^{2}$-norm for the unknowns $\omega$ and $p$ and in the $H^{1}$-norm for $\left(u_{1}, u_{2}\right)$. These errors are computed for $\beta=0.1$. Note that these errors are calculated between the numerical approximation and an interpolate of the exact solution $\left(P_{0}\right.$-interpolation for $\omega$ and $p$, and $P_{1}$-interpolation for $\left.\mathbf{u}\right)$. In this example, we have meshed the domain $\Omega$ using different structured triangulations.

We notice that the $O(h)$ behavior of the error is in agreement with the theoretical error bounds: the error is divided by 2 when the mesh size $h$ is divided by 2 . A comparison between the exact solution and the computed one is depicted in Figures 1 2 and 3. This comparison is performed with an unstructured mesh using 1992 elements and 1022 nodes.

Next, we investigate the behavior of the error for different values of the parameter $\beta$ using the previous mesh. Table 2 tends to indicate that $\beta$ should be chosen neither too large nor too small. Indeed, an optimal value for $\beta$ seems to be around 0.1.

Tests 3 and 4: Pipe flow with an obstacle and T-shape. In the following two numerical experiments we consider more general domains and boundary conditions. In the first one, we simulate a pipe flow with an obstacle. In the second experiment we consider the problem of a $\mathrm{T}$-shape bifurcation. In both simulations, the pressure is imposed on the inlet and outlet boundaries of the domain together with $\mathbf{u} \cdot \mathbf{t}$. We choose $\mathbf{u}=\mathbf{0}$ on the other boundaries. In the pipe flow example the difference between the pressures on the inlet and outlet boundaries is equal to 4 . In the Tshape test, the difference between the pressures on the inlet and on the right-hand side outlet is equal to 4 , while the pressure difference on the inlet and on the lefthand side outlet is equal to 2. As in the previous example, we present in Figures 6]11 the velocity field, the vorticity $\omega$ and the pressure. 

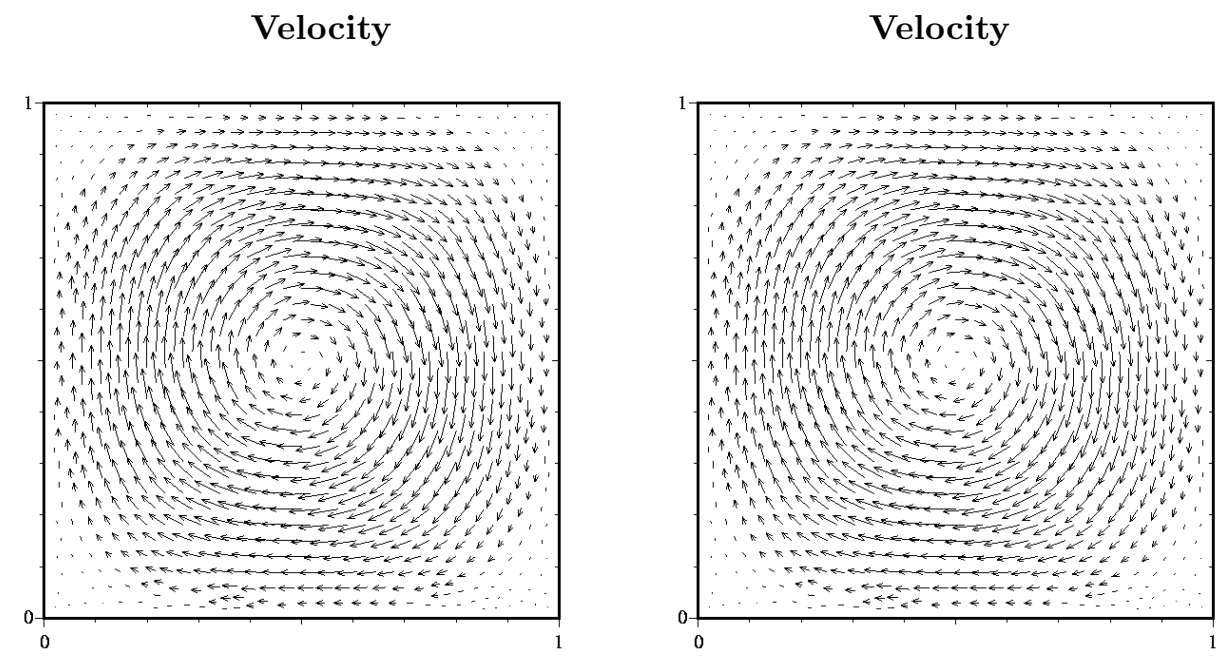

Figure 1. Exact and calculated velocities

Vorticity

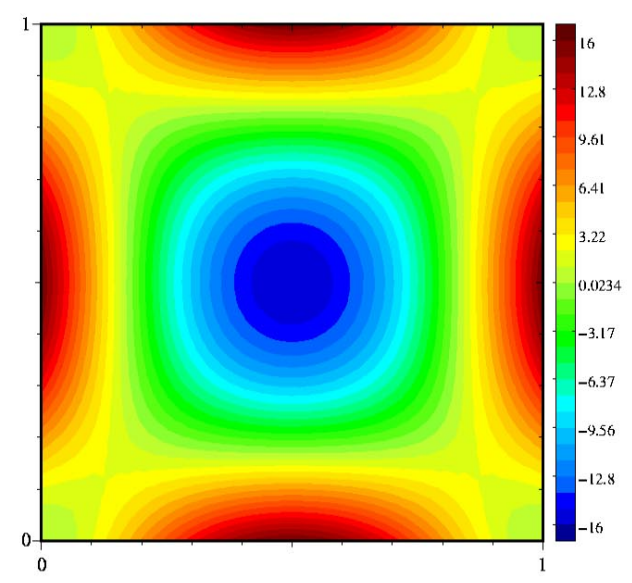

Pressure

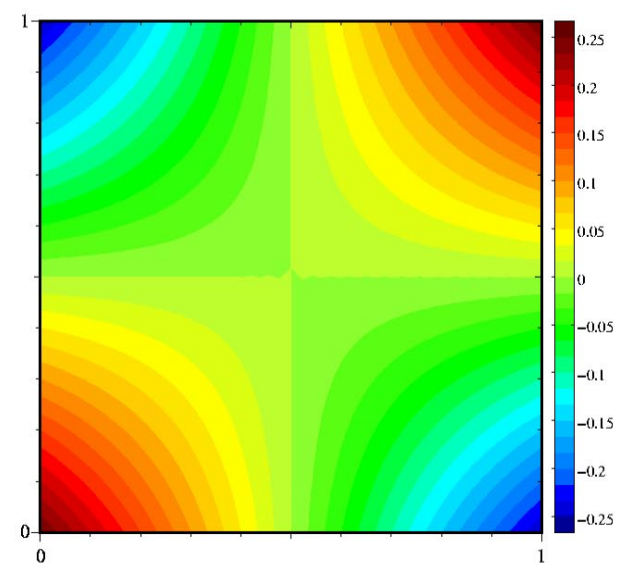

FIGURE 2. Exact vorticity and pressure

Test 2: The cavity test. Now we consider more realistic examples. The first one is the cavity test. The domain $\Omega$ and the mesh are the same as in the preceding example. The right-hand sides are equal to zero, $\mathbf{u}=(1,0)$ on the upper boundary, and $\mathbf{u}=\mathbf{0}$ on the three other sides of $\Omega$. Figures 4 and 5 depict the numerical results and demonstrate the good behavior of the method.

Test 5: The step. The last numerical results we present in this paper are obtained for the so-called step test. We impose a Poiseuille flow $(\mathbf{u} \neq \mathbf{0}$ is given $)$ on the inlet and the outlet boundaries, and we take $\omega=0$ together with $\mathbf{u} \cdot \mathbf{n}=0$ on the upper boundary. The results are presented in Figures 12-14. 
Vorticity

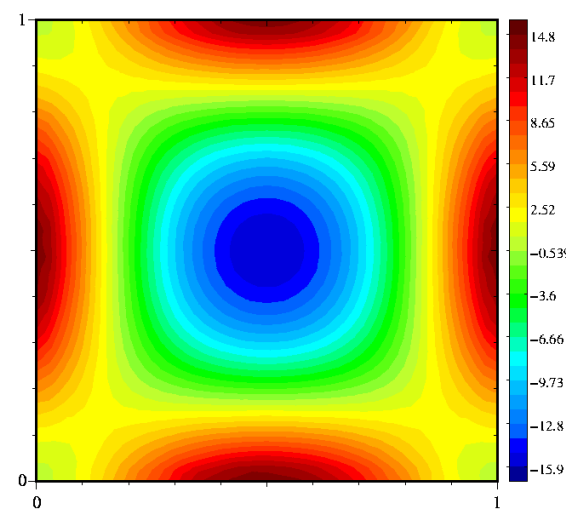

Pressure

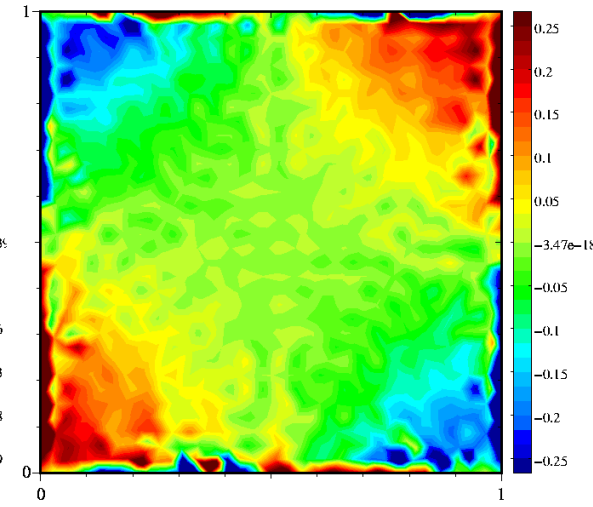

Figure 3. Calculated vorticity and pressure

Velocity

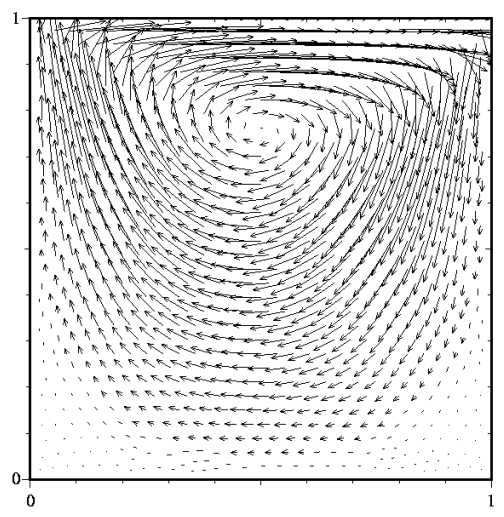

Figure 4. Calculated velocity for the cavity test
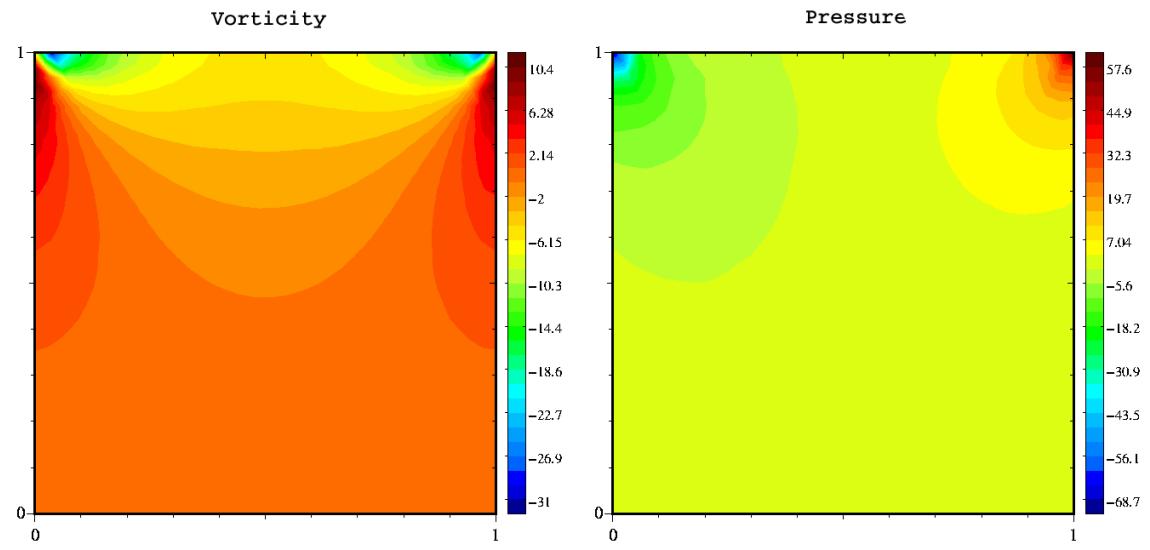

Figure 5. Vorticity and pressure for the cavity test 
mesh : 1506 triangles and 819 nodes

\section{Velocity}

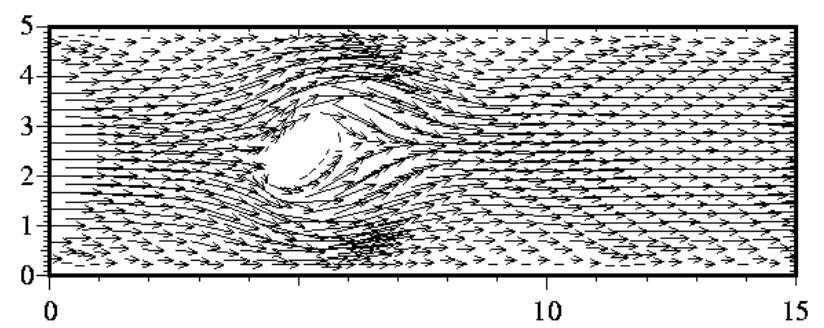

Figure 6. Calculated velocity for the pipe flow test Vorticity

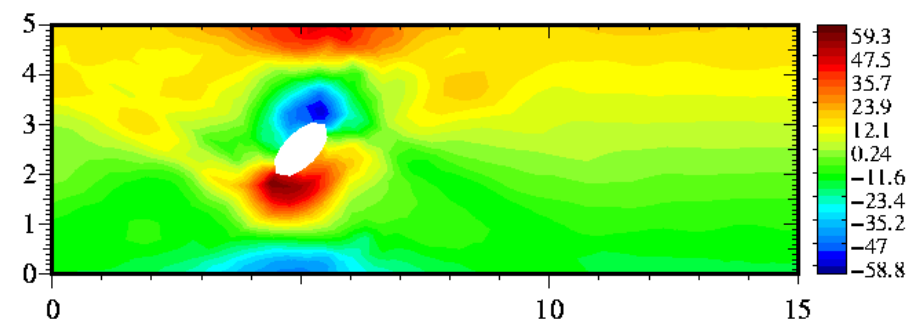

Figure 7. Calculated vorticity for the pipe flow test

Pressure

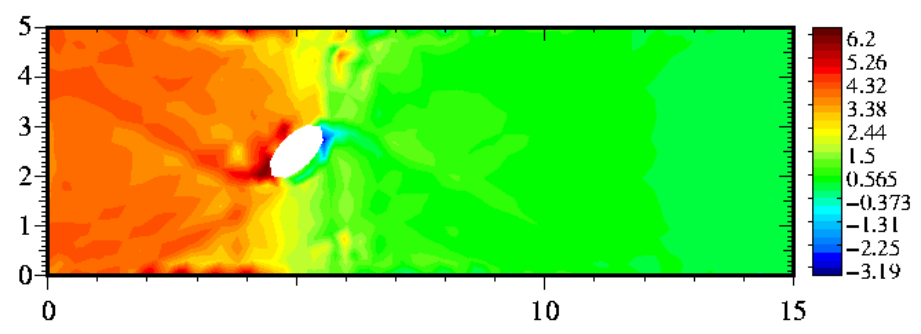

Figure 8. Calculated pressure for the pipe flow test 
mesh: 3150 triangles and 1696 nodes

\section{Velocity}

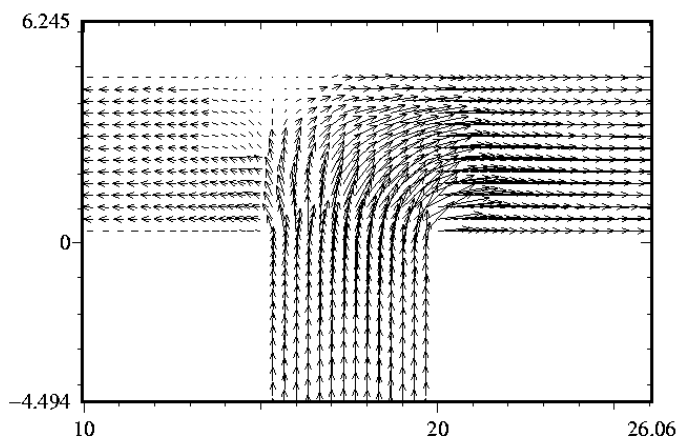

Figure 9. Calculated velocity for the T-shape test

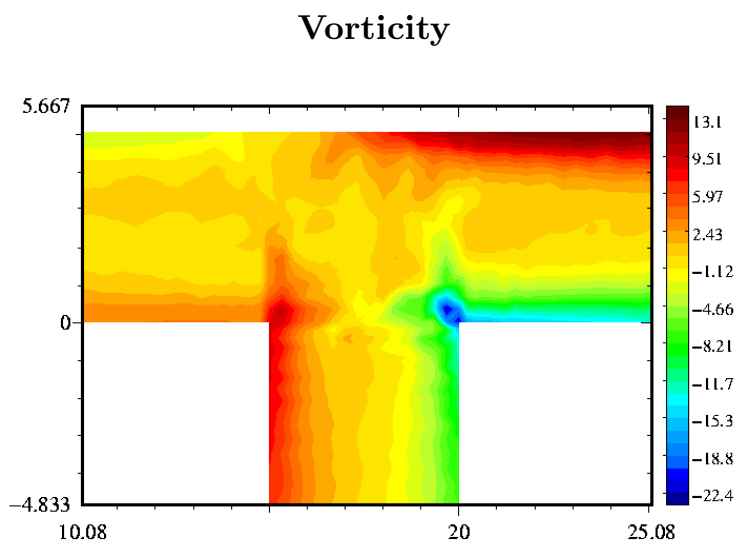

Figure 10. Calculated vorticity for the T-shape test Pressure

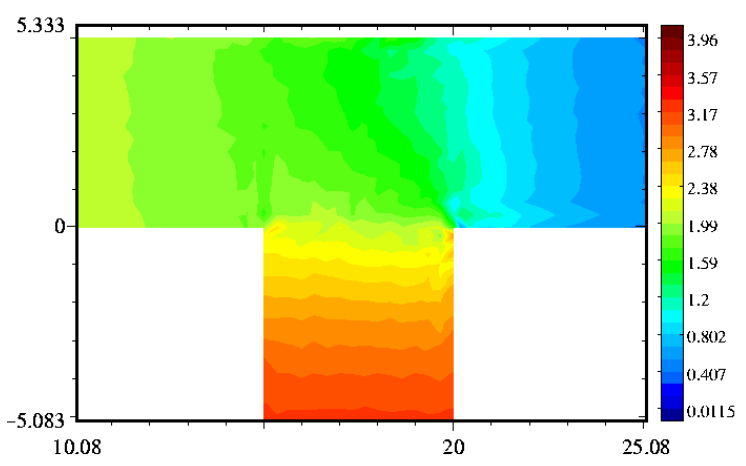

Figure 11. Calculated pressure for the T-shape test 


\section{Velocity}

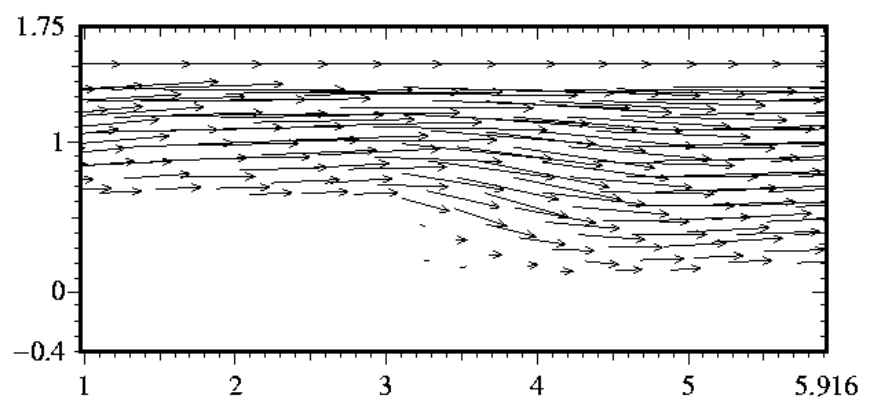

Figure 12. Calculated velocity for the step test Vorticity

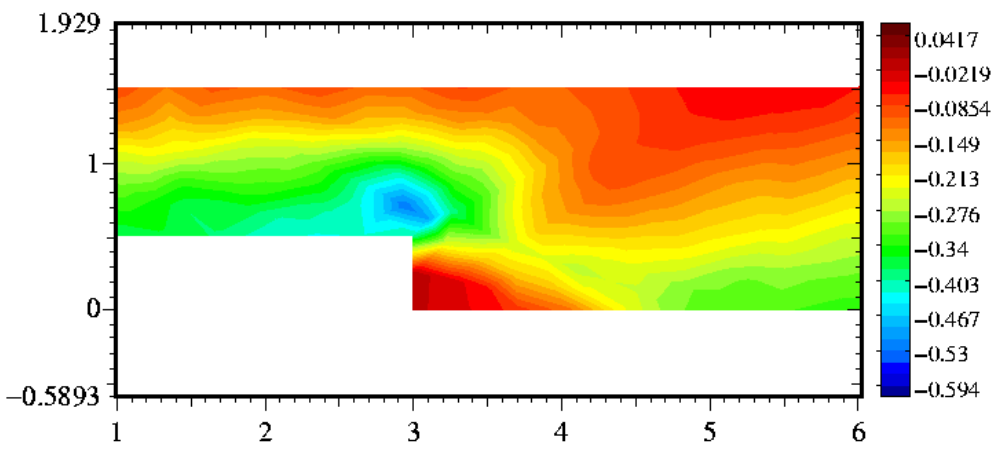

FIgURE 13. Calculated vorticity for the step test

Pressure

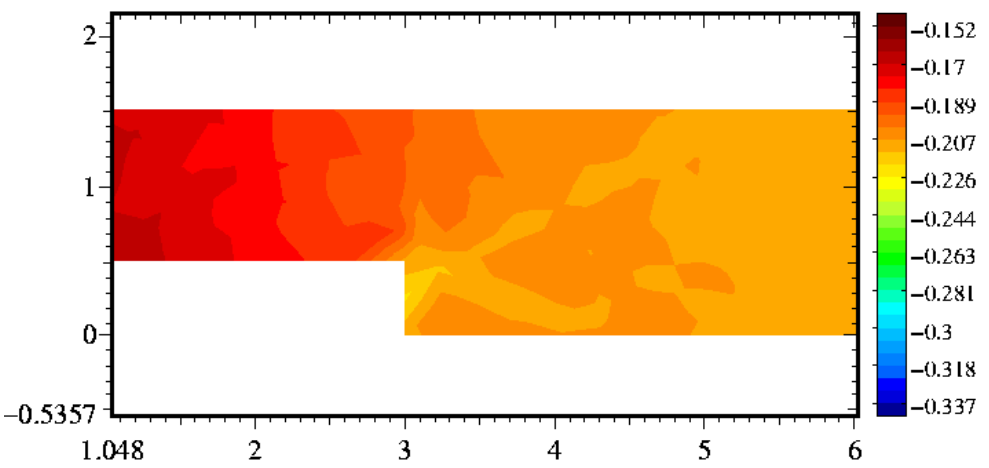

Figure 14. Calculated pressure for the step test 


\section{REFERENCES}

[1] Amara, M. and Bernardi, C., Convergence of a finite element discretization of the NavierStokes equations in vorticity and stream function formulation, Mathematical Modelling and Numerical Analysis, vol. 33, n. 5, pp. 1033-1056 (1999). MR 2000k:65200

[2] Amara, M. and El Dabaghi, F., An optimal $C^{0}$ finite element algorithm for the $2 D$ biharmonic problem: Theoretical analysis and numerical results, Numerische Mathematik, vol. 90, n. 1, pp. 19-46 (2001). MR 2002h:65172

[3] Amara, M., Chacon Vera, E. and Trujillo, D., Stokes equations with non standard boundary conditions, Prpubli. du Labo. de Math. Appli. de Pau, n. 13, (2001).

[4] Bernardi, C. and Girault, V., A local regularization operator for triangular and quadrilateral finite elements, SIAM J. Numer. Anal., vol. 35, n. 5, pp. 1893-1916 (1998). MR 99g:65107

[5] Bernardi, C., Girault, V. and Maday, Y., Mixed spectral element approximation of the NavierStokes equation in the stream function and vorticity formulation, IMA, J. of Numer. Anal, 12, pp. 564-608, 1992. MR 93i:65113

[6] Costabel, M., A remark on the regularity of solutions of Maxwell's equations on Lipschitz domains, Mathematical Methods in the Applied Sciences, vol. 12, 365-368 (1990). MR 91c:35028

[7] Conca, C., Pares, C., Pironneau, O. and Thiriet, M., Navier-Stokes equations with imposed pressure and velocity fluxes, International Journal for Numerical Methods in Fluids, vol. 20, 267-287 (1995). MR 96d:76019

[8] Dubois, F., Salaün M. and Salmon S., Discrete harmonics for stream function-vorticity Stokes problem, Numer. Math., vol. 92, 711-742 (2002). MR 2003h:76088

[9] John, F., Partial Differential Equations, Applied Mathematical Sciences, 1. Springer-Verlag, (1978). MR 80f:35001

[10] Girault, V. and Raviart, P.-A., Finite Element Methods for the Navier-Stokes Equations. Theory and Algorithms, Springer-Verlag, (1986). MR 88b:65129

[11] Grisvard, P., Elliptic Problems in Nonsmooth Domains, Pitman, (1985). MR 86m:35044]

[12] Hughes, T. and Franca, L., A new finite element formulation for computational fluid dynamics. VII. The Stokes problem with various well-posed boundary conditions: Symetric formulations that converge for all velocity/pressure space, Comput. Methods in Appl. Mech. and Eng., 65, 85-96, (1987). MR 89j:76015g

[13] Lions, J.L. and Magenes, E., Problèmes aux limites non homogènes et applications, vol 1, Dunod, Paris (1969). MR 40:512

IPRA-LMA, Université de PaU, 64000 PaU, France

E-mail address: mohamed.amara@univ-pau.fr

IPRA-LMA, Université de PAU, 64000 PAU, France

E-mail address: david.trujillo@univ-pau.fr

Departamento de Ecuaciones Diferenciales y Análisis, Universidad de Sevilla, 41080 Sevilla, Spain

E-mail address: eliseo@numer.us.es 